Pacific

Journal of

Mathematics

PROPERLY EMBEDDED MINIMAL DISKS BOUNDED BY NONCOMPACT POLYGONAL LINES

LeONor Ferrer and Francisco MarTín

Volume $214 \quad$ No. 1

March 2004 


\title{
PROPERLY EMBEDDED MINIMAL DISKS BOUNDED BY NONCOMPACT POLYGONAL LINES
}

\author{
LEONOR FERRER AND FRANCISCO MARTÍN
}

\begin{abstract}
In this paper we give a uniqueness and existence result for minimal disks with some noncompact, U-shaped boundaries in a slab of $\mathbb{R}^{3}$.
\end{abstract}

\section{Introduction and preliminaries.}

Minimal surfaces containing straight lines have special properties that distinguish them from the rest of minimal surfaces. In this article, we emphasize Schwarz's reflection principle. Examples of this type were well studied during the last two centuries.

Recently, in [11], F.J. López and F. Wei obtained an existence and uniqueness theorem for properly immersed minimal disks whose boundaries consist of two disjoint straight lines and a segment which meets the lines orthogonally.

Following this, López and the second author of this paper have constructed a deformation of López-Wei disks which consists of properly embedded minimal disks bounded by straight lines and contained in a wedge of a slab (see [9] and [10]). Essentially, the deformation modifies the angle formed by the two halfplanes containing the connected components of the boundary. The surfaces that appear in this deformation for angle zero correspond to some Jenkins-Serrin graphs (see [6]). The López-Martín examples have nice geometric properties such as the convex hull property. These examples are a solution to Plateau's problem for a polygonal noncompact boundary consisting of a double $\mathbf{U}$ shaped contour (see Figure 1). These surfaces can be used as a new type of barrier for the maximum principle application ([8] and [9]). Examples of this kind are also closely related to minimal surfaces with helicoidal ends $([\mathbf{1 5}])$.

In this paper, we obtain all the solutions to the aforementioned Plateau problem with noncompact polygonal boundary, which are contained in the slab, but not lie necessarily in the convex hull of their boundary (see Figure 2). To be more precise, we deal with the study of properly embedded minimal surfaces whose boundary $\Gamma_{\theta d}$ consists of the following configuration of straight lines:

Fix $\theta \in[0, \pi]$ and $d \geq 0$, and consider two half-lines $r_{1}^{+}$and $r_{1}^{-}$in $\mathbb{R}^{3}$, meeting at an angle of $\theta$. If $\theta=0$ this means that the straight lines are 
parallel. Let $q_{1}^{+}$and $q_{1}^{-}$be two points in $r_{1}^{+}$and $r_{1}^{-}$, respectively, such that they are symmetric with respect the inner bisector of these half-lines. We choose $q_{1}^{+}$and $q_{1}^{-}$in such a way that either $q_{1}^{+}=q_{1}^{-}$or the half-lines $\ell_{1}^{+}$and $\ell_{1}^{-}$on $r_{1}^{+}$and $r_{1}^{-}$starting at $q_{1}^{+}$and $q_{1}^{-}$, respectively, do not intersect. Write $d=\operatorname{dist}\left(q_{1}^{+}, q_{1}^{-}\right)$.

Let $\Pi_{1}$ be the plane determined by $\ell_{1}^{+}$and $\ell_{1}^{-}, \Pi_{2}$ a plane parallel and distinct to $\Pi_{1}$ and let $S$ denote the slab determinated by $\Pi_{1}$ and $\Pi_{2}$. Let $\ell_{2}^{+}$and $\ell_{2}^{-}$be the orthogonal projections to $\Pi_{2}$ of $\ell_{1}^{+}$and $\ell_{1}^{-}$, respectively. Denote $q_{2}^{+}$(resp. $q_{2}^{-}$) as the orthogonal projection to $\Pi_{2}$ of $q_{1}^{+}$(resp. $q_{1}^{-}$), and label $\ell_{0}^{+}$(resp. $\left.\ell_{0}^{-}\right)$as the segment $\left[q_{1}^{+}, q_{2}^{+}\right]$(resp. $\left.\left[q_{1}^{-}, q_{2}^{-}\right]\right)$. Finally, we define

$$
\Gamma_{\theta d}^{+}=\bigcup_{i=0}^{2}\left(\ell_{i}^{+}\right), \quad \Gamma_{\theta d}^{-}=\bigcup_{i=0}^{2}\left(\ell_{i}^{-}\right), \quad \Gamma_{\theta d}=\Gamma_{\theta d}^{+} \cup \Gamma_{\theta d}^{-} .
$$

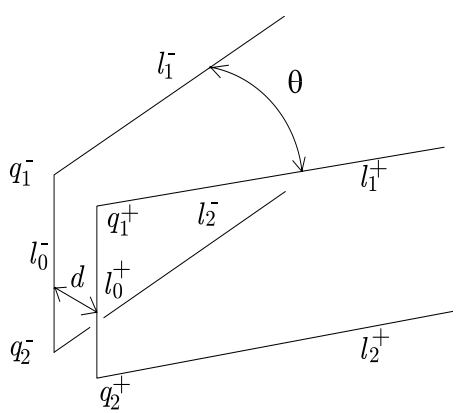

Figure 1. The curve $\Gamma_{\theta d}$.

We consider the following generalized Plateau problem:

Problem 1. Determine a properly immersed minimal surface $X: M \rightarrow \mathbb{R}^{3}$ satisfying:

(1) $M$ is homeomorphic to the closed unit disk $\overline{\mathbb{D}}$ minus two boundary points $E_{1}$ and $E_{2}$, that we call the ends of $M$.

(2) $X(\partial(M))=\Gamma_{\theta d}$.

(3) If $d>0, X$ is an embedding.

(4) In the limit case $\ell_{0}^{+}=\ell_{0}^{-}$(i.e., $d=0$ ), the maps $\left.X\right|_{M-\gamma^{+}}$and $\left.X\right|_{M-\gamma^{-}}$ are injective, where $\gamma^{+}$and $\gamma^{-}$are the two connected components of $\partial(M)$.

(5) $X(M)$ lies in a slab that contains $S$.

Observe that if (5) is satisfied then it is easy to prove (see Lemma 2.1 in [12]) that $X(M)$ lies in the slab $S$. Then Condition (5) is equivalent to

(5) $X(M)$ lies in $S$.

We prove the following: 
Main Theorem. If $0<\theta<\pi$ there exist $d_{\theta}$ and $d_{\theta}^{\prime}$ with $0<d_{\theta}^{\prime}<d_{\theta}$ such that:

(i) If $d>d_{\theta}$ there are no solutions of Problem 1.

(ii) If $d=d_{\theta}$, Problem 1 has a unique solution.

(iii) If $d \in] d_{\theta}^{\prime}, d_{\theta}[$ or $d=0$, Problem 1 has two solutions.

(iv) If $d=d_{\theta}^{\prime}$, Problem 1 has three solutions.

(v) If $d \in] 0, d_{\theta}^{\prime}[$, Problem 1 has four solutions.

If $\theta=\pi$ there exist $d_{\pi}$ with $0<d_{\pi}$ such that:

(i) If $d>d_{\pi}$ there are no solutions of Problem 1.

(ii) If $d=0$ or $d=d_{\pi}$, Problem 1 has a unique solution.

(iii) If $d \in] 0, d_{\pi}[$, Problem 1 has two solutions.

If $\theta=0$ there exist $d_{0}^{\prime}$ with $0<d_{0}^{\prime}<\operatorname{dist}\left(\Pi_{1}, \Pi_{2}\right)$ such that:

(i) If $d \geq \operatorname{dist}\left(\Pi_{1}, \Pi_{2}\right)$ there are no solutions of Problem 1.

(ii) If $d=0$ or $d \in] d_{0}^{\prime}$, dist $\left(\Pi_{1}, \Pi_{2}\right)[$, Problem 1 has a unique solution.

(iii) If $d=d_{0}^{\prime}$, Problem 1 has two solutions.

(iv) If $d \in] 0, d_{0}^{\prime}[$, Problem 1 has three solutions.

López and Wei proved in [11] that there exists a unique solution of Problem 1 when $\theta=\pi$ and $d=0$. Therefore, we always omit this case in our discussions.
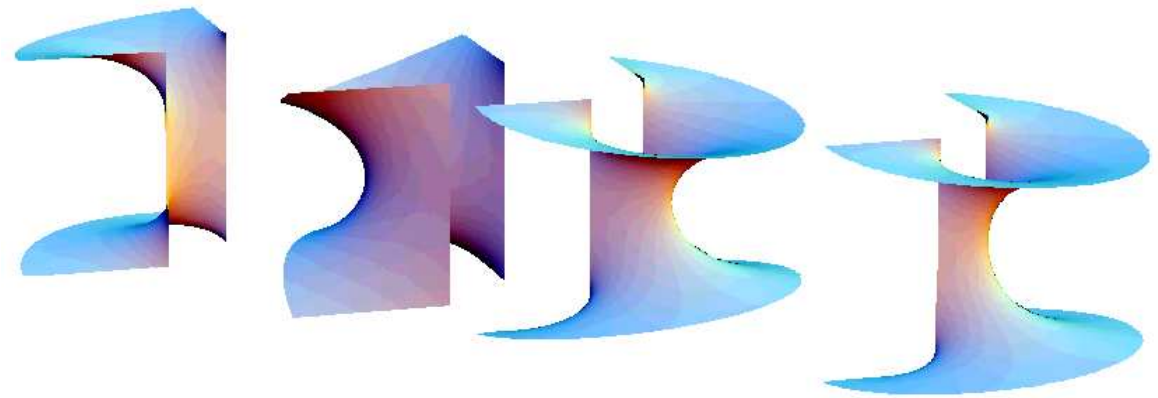

Figure 2. The four solutions in case $\theta=\frac{\pi}{2}, d=\frac{\operatorname{dist}\left(\Pi_{1}, \Pi_{2}\right)}{2}$. The first and second one on the left corresponds to LópezMartín examples.

The aim of this paper is to prove the uniqueness and existence of the solutions stated in the Main Theorem. The paper is set out as follows:

In Section 2, we obtain the uniqueness result stated in the above theorem. For the sake of clarity we divide the proof in several subsections. In the first one, we shall see that if $M$ is a solution of our Plateau problem then $M$ is conformally equivalent to a twice punctured closed disk with piecewise analytic boundary and its meromorphic data extend to the closed disk. 
Among the results obtained in this subsection, we emphasize the following proposition:

Proposition 1. Assume $X: M \longrightarrow \mathbb{R}^{3}$ is a solution of Problem 1 for $0 \leq \theta<\pi$ and denote by $\mathcal{E}\left(\Gamma_{\theta d}\right)$ the convex hull of $\Gamma_{\theta d}$. Then $X(M)$ lies either in $\mathcal{E}\left(\Gamma_{\theta d}\right)$ or in $\left(S \backslash \mathcal{E}\left(\Gamma_{\theta d}\right)\right) \cup \Gamma_{\theta d}$. If $\theta=\pi$, then $X(M)$ lies in one of the half-slabs determinated by the strip $\mathcal{E}\left(\Gamma_{\pi d}\right)$.

Roughly speaking, the above proposition asserts that if $0 \leq \theta<\pi$, then the solutions of our problem lie either in the interior of the convex hull of the boundary or in the exterior of it. Subsection 2.2 is devoted to proving that $M$ inherits the horizontal symmetry of its boundary and also the vertical symmetry in case $d=0$. Finally, in Subsection 2.3, taking into account the preceding steps, we determine a model of the complex structure and Weierstrass representation of any solution of Plateau's problem above. As a consequence, we obtain that, in the general case, a solution of our Plateau problem also inherits the vertical symmetry of its boundary.

The existence part of the Main Theorem can be found in Section 3. We prove that the Weierstrass data obtained in Section 2 really correspond to solutions of our problem.

As we mentioned before, López-Martín examples can be used as barriers in order to prove nonexistence results for minimal surfaces with planar boundaries in a wedge of a slab. Furthermore, they extended the family of minimal surfaces satisfying the convex hull property. To state these results we need some notation. Define $L=\left\{(0,0, t) \mid-\frac{1}{2}<t<\frac{1}{2}\right\}$ and $W=\left\{\left(x_{1}, x_{2}, x_{3}\right) \in \mathbb{R}^{3} \mid-\frac{1}{2} \leq x_{3} \leq \frac{1}{2}\right\}$. For $\theta \in[0, \pi]$, we also write $W_{\theta}=L \cup\left\{\left(x_{1}, x_{2}, x_{3}\right) \in W \backslash L \mid \operatorname{Arg}\left(\left(x_{1}, x_{2}\right)\right) \in[0, \theta]\right\}$ and $\Sigma_{\theta}=L \cup$ $\left\{\left(x_{1}, x_{2}, x_{3}\right) \in W_{\pi} \backslash L \mid \operatorname{Arg}\left(\left(x_{1}, x_{2}\right)\right)=\theta\right\}$. Using this notation López and the second author have proved the following:

Theorem 1 ([10]). Let $M$ be a connected properly immersed minimal surface in a wedge $W_{2 \pi-\epsilon}$ for some $0<\epsilon<2 \pi$. Then one has:

(i) If $\partial(M) \subset \Sigma_{0}$, then $M$ is a planar region in $\Sigma_{0}$.

(ii) If $\partial(M) \subset W_{\theta}$, for $\left.\theta \in\right] 0, \pi[$, then $M$ lies in the convex hull of its boundary.

Finally, we would like to mention that the Main Theorem and Proposition 1 are announced in the proceedings of the conference Differential Geometry Valencia 2001 ([5]).

\section{Conformal structure and Weierstrass representation.}

As we mentioned before, this section is devoted to study the underlying complex structure and Weierstrass data of the solutions of our problem.

Throughout this paper $\left(x_{1}, x_{2}, x_{3}\right)$ denotes a set of Cartesian coordinates such that: 
- $\ell_{0}^{+}$and $\ell_{0}^{-}$have the direction of $x_{3}$-axis,

- $\left\{x_{3}=\frac{1}{2}\right\}$ and $\left\{x_{3}=-\frac{1}{2}\right\}$ are the equations of planes $\Pi_{1}$ and $\Pi_{2}$, respectively,

- the origin is the middle point between $\frac{q_{1}^{+}+q_{2}^{+}}{2}$ and $\frac{q_{1}^{-}+q_{2}^{-}}{2}$,

- the $x_{2}$-axis is the inner bisector of the orthogonal projection of $\ell_{i}^{+}$and $\ell_{i}^{-}$to the plane $x_{3}=0, i=1,2$, and

- $\Gamma_{\theta d}^{+} \subset\left\{x_{1} \geq 0\right\}$.

Along this section, $X: M \longrightarrow \mathbb{R}^{3}$ denote a proper conformal minimal immersion satisfying Conditions (1)-(5) of Problem 1. For the sake of simplicity, we use $\Gamma, \Gamma^{+}$and $\Gamma^{-}$instead of $\Gamma_{\theta d}, \Gamma_{\theta d}^{+}$and $\Gamma_{\theta d}^{-}$. Taking into account that $X(M) \subset S$ and the maximum principle, we deduce that $\Pi_{i} \cap X(M) \subset \Pi_{i} \cap \Gamma$, for $i=1,2$.

As we announced, we shall divide the study of conformal structure in several subsections.

2.1. Conformal type of $\boldsymbol{M}$. The conformal type of $M$ can be easily determined using a global result on conformal structure of properly immersed minimal surfaces by P. Collin, R. Kusner, W.H. Meeks and H. Rosenberg (see [4]). From Theorem 3.1 of [4] we obtain that $M$ is parabolic and hence, taking into account the topological type of $M, M$ is conformally equivalent to the closed unit disk $\overline{\mathbb{D}}$ minus two boundary points $E_{1}$ and $E_{2}$, where the biholomorphism extends piecewise analytically to the boundary.

Next, we prove that the Gauss map and Weierstrass data extend continuously to the ends. To obtain this, we need some additional results.

Let $U\left(E_{i}\right), i=1,2$, be two open disjoint neighbourhoods of the ends of $M$ and let $C_{a}$ denote the catenoid given by the equation $x_{1}^{2}+x_{2}^{2}=a^{2} \cosh ^{2}\left(\frac{x_{3}}{a}\right)$, where $a \in \mathbb{R}^{+}$. Define $\sigma_{a}=X^{-1}\left(X(M) \cap C_{a}\right)$, for $a>0$. With this notation we shall prove the following:

Lemma 1. There exists $a_{0}>0$ such that for $a \geq a_{0}, \sigma_{a}=\sigma_{a}^{1} \cup \sigma_{a}^{2}$, where $\sigma_{a}^{1}$ and $\sigma_{a}^{2}$ are two disjoint simple compact analytic curves such that $\sigma_{a}^{i} \subset U\left(E_{i}\right)$, for $i=1,2$.

Proof. Clearly, since $X(M) \cap C_{a}$ is compact and $X$ is proper, we have that $\sigma_{a}$ is compact for $a>0$. Furthermore, $\sigma_{a}$ is a set of properly immersed analytic lines, because it is the intersection of distinct minimal surfaces. Denote by $\operatorname{Int}\left(C_{a}\right)$ and $\operatorname{Ext}\left(C_{a}\right)$, the interior and exterior connected component of $\mathbb{R}^{3} \backslash C_{a}$, respectively.

Note that we can consider $a_{1}$ sufficiently large to insure the following: $\ell_{0}^{+} \cup \ell_{0}^{-} \subset \operatorname{Int}\left(C_{a}\right)$ and $S \cap \operatorname{Int}\left(C_{a}\right) \subset S \cap \operatorname{Int}\left(C_{a^{\prime}}\right)$ if $a_{1} \leq a \leq a^{\prime}$. As $C_{a}$ and $X(M)$ are transverse along $\bigcup_{i=1}^{2} \ell_{i}^{+} \cup \ell_{i}^{-}$, for $a \geq a_{1}$, we can assert that only one curve lying in $\sigma_{a}$ approaches to each one of the four points in $\sigma_{a} \cap \partial(M)$. 
Moreover, since $\sigma_{a_{1}}$ is compact, we can find $U^{\prime}\left(E_{i}\right)$, connected neighbourhoods of $E_{i}$ such that $U^{\prime}\left(E_{i}\right) \subset U\left(E_{i}\right)$ and $X\left(U^{\prime}\left(E_{i}\right)\right) \subset \operatorname{Ext}\left(C_{a_{1}}\right)$. Consider now $a_{0}>a_{1}$ such that $\sigma_{a_{0}} \subset U^{\prime}\left(E_{1}\right) \cup U^{\prime}\left(E_{2}\right)$.

Therefore, if $a \geq a_{0}$ we deduce that $\sigma_{a}=\sigma_{a}^{1} \cup \sigma_{a}^{2}$, with $\sigma_{a}^{i} \subset U^{\prime}\left(E_{i}\right)$, $i=1,2$ and $\sigma_{a}^{1} \cap \sigma_{a}^{2}=\emptyset$. Now, we must prove that $\sigma_{a}^{i}$ are simple curves for $i=1,2$. Suppose that there exists a disk $\Omega$ in $U^{\prime}\left(E_{i}\right)$ bounded by an arc of $\sigma_{a}^{i}$. In this case, either $X(\Omega) \subset \operatorname{Ext}\left(C_{a}\right)$ or $X(\Omega) \subset \operatorname{Int}\left(C_{a}\right)$. In the first case, we have that $X(\Omega) \subset \operatorname{Ext}\left(C_{a}\right) \cap \operatorname{Int}\left(C_{a_{2}}\right)$, for some $a \leq a_{2}$. Hence, using the family of catenoids $\left\{C_{t}\right\}_{a \leq t \leq a_{2}}$ and the maximum principle, we obtain that $X(\Omega)$ is contained in the catenoid $C_{a}$, which is contrary to our assumptions. Moreover, since $a_{0} \leq a$ we can assert that $X(\Omega) \subset \operatorname{Ext}\left(C_{a_{1}}\right)$. Consequently, if $X(\Omega) \subset \operatorname{Int}\left(C_{a}\right)$ we may consider the family of catenoids $\left\{C_{t}\right\}_{a_{1} \leq t \leq a}$. The maximum principle gives again a contradiction.

Label $\gamma_{i}^{+}=X^{-1}\left(\Gamma_{i}^{+}\right)$and $\gamma_{i}^{-}=X^{-1}\left(\Gamma_{i}^{-}\right)$, for $i=1,2$. Consider also $\gamma_{0}^{+}=X^{-1}\left(\ell_{0}^{+} \cup \ell_{0}^{-}\right) \cap \gamma^{+}$and $\gamma_{0}^{-}=X^{-1}\left(\ell_{0}^{+} \cup \ell_{0}^{-}\right) \cap \gamma^{-}$.

Concerning the boundary behaviour we have, up to relabellings, three possibilities:

Case 1. $X\left(\gamma^{+}\right)=\Gamma^{+}, X\left(\gamma^{-}\right)=\Gamma^{-}$and $\gamma_{i}^{+} \cup \gamma_{i}^{-}$diverges to $E_{i}$, for $i=1,2$ (see Figure 3.(1)).

Case 2. $X\left(\gamma^{+}\right)=\Gamma^{+}, X\left(\gamma^{-}\right)=\Gamma^{-}, \gamma_{1}^{+} \cup \gamma_{2}^{-}$diverges to $E_{1}$ and $\gamma_{2}^{+} \cup \gamma_{1}^{-}$ diverges to $E_{2}$ (see Figure 3.(2)).

Case 3. When $d=0$ we have also the case $X\left(\gamma^{+}\right)=\ell_{1}^{+} \cup \ell_{0} \cup \ell_{2}^{-}, X\left(\gamma^{-}\right)=$ $\ell_{1}^{-} \cup \ell_{0} \cup \ell_{2}^{+}$and $\gamma_{i}^{+} \cup \gamma_{i}^{-}$diverges to $E_{i}$, for $i=1,2$, where $\ell_{0}=\ell_{0}^{+}=\ell_{0}^{-}$ (see Figure 3(3)).

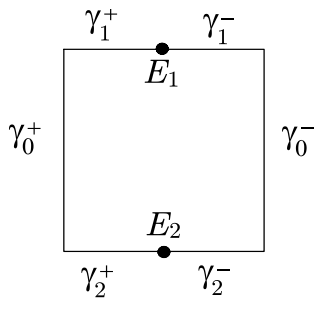

(1)

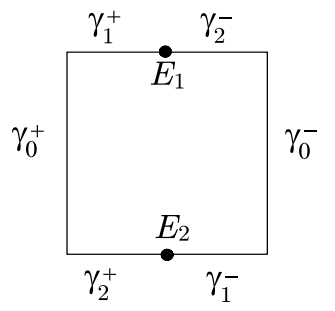

(2)

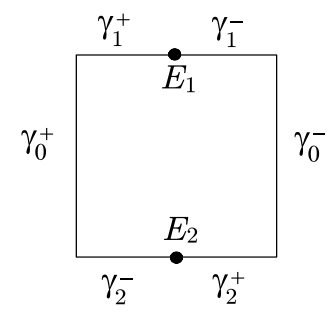

(3)

\section{Figure 3.}

Now, we shall prove that if $d=0$ then Case 2 and Case 3 do not occur.

Lemma 2. Assume $d=0$. Then the boundary of $X: M \longrightarrow \mathbb{R}^{3}$ is as in Case 1.

Proof. Note that if $d=0$ and the boundary is either as in Case 2 or as in Case 3, $X(M)$ contains a Möbius strip. Let us define $t_{\rho}$ as the translation of vector $(0,0, \rho)$ and consider $N$ the topological surface of $\mathbb{R}^{3}$ given by 
$N=\bigcup_{n \in \mathbb{Z}} t_{n}(X(M))$. Since $X(M) \subset S$ we have that $N$ is a connected embedded topological surface in $\mathbb{R}^{3}$. A well-known topological result asserts that then $N$ must be orientable, but this is absurd because $N$ contains Möbius strips.

Denote $\vec{a}_{2}=(0,1,0)$. Observe that $\vec{a}_{2}$ is the unitary vector in the direction of the inner bisector of $\ell_{2}^{+}$and $\ell_{2}^{-}$pointing to $\mathcal{E}(\Gamma)$. At this point we can prove a restatement of Proposition 1 above:

Proposition 1. If $0 \leq \theta<\pi$, then $X(M)$ lies either in $\mathcal{E}(\Gamma)$ or in $(S \backslash$ $\mathcal{E}(\Gamma)) \cup \Gamma$. If $\theta=\pi$, then $X(M)$ lies in one of the half-slabs determinated by the strip $\mathcal{E}(\Gamma)$.

Proof. Assume $0 \leq \theta<\pi$. In accordance to Lemma 2 we have that the boundary behaviour is either as in Case 1 or as in Case 2. Consider $\beta=$ $X^{-1}\left(X(M) \cap\left\{x_{2}=0\right\}\right)$. Since $\beta$ is a nodal set of an harmonic function we have that $\beta$ is a set of properly immersed analytic lines. Using the maximum principle we obtain that there are no compact connected regions of $M$ bounded by curves in $\beta$. Furthermore, as we are assuming $0 \leq \theta<\pi$, the theorem of the order of contact (see $[\mathbf{1 3}, \S 437]$ ) gives us that there are no curves in $\beta$ approaching to either $\gamma_{i}^{+}$or $\gamma_{i}^{-}$, for $i=1,2$. Now, we consider the following half-strips:

$$
\begin{aligned}
& B^{+}=\left\{\left(x_{1}, 0, x_{3}\right) \mid x_{1} \geq \frac{d}{2}, \frac{-1}{2} \leq x_{3} \leq \frac{1}{2}\right\}, \\
& B^{-}=\left\{\left(x_{1}, 0, x_{3}\right) \mid x_{1} \leq \frac{-d}{2}, \frac{-1}{2} \leq x_{3} \leq \frac{1}{2}\right\} .
\end{aligned}
$$

First, we shall prove that if there exists a curve in $\beta$ starting at either $\gamma_{0}^{+}$or $\gamma_{0}^{-}$and diverging to one end, then there are no curves starting at the same vertical segment and diverging to the same end. Assume that $\beta^{\prime}$ and $\beta^{\prime \prime}$ are two curves starting at $\gamma_{0}^{+}$and diverging to $E_{1}$. The other cases can be treated in the same way. Clearly, if $d>0$ we have $X\left(\beta^{\prime}\right) \cup X\left(\beta^{\prime \prime}\right) \subset B^{+}$. Suppose that $d=0$ and $X\left(\beta^{\prime}\right)$ and $X\left(\beta^{\prime \prime}\right)$ are contained in different halfstrips. Then we can consider, taking a piece of $\gamma_{0}^{+}$if necessary, a piecewise analytic curve $\hat{\beta}$ that diverge to $E_{1}$ and contains $\beta^{\prime}$ and $\beta^{\prime \prime}$. It is not difficult to see that there is an angle between the curves in $\hat{\beta}, \Theta$, that goes by $X$ to an angle greater or equal than $2 \Theta$. Since $X: M \longrightarrow \mathbb{R}^{3}$ is conformal this is a contradiction. Then we conclude that in both cases $X\left(\beta^{\prime}\right)$ and $X\left(\beta^{\prime \prime}\right)$ are contained in the same half-strip. Therefore, we can find a connected component, $\Omega$, of $M \backslash \beta$ such that $X(\Omega)$ is contained in one of the half-slabs determinated by $\left\{x_{2}=0\right\}$ and $X(\partial(\Omega))$ is in a half-strip. Consequently, applying Statement (i) in Theorem 1 we obtain that $X(\Omega)$ is a planar domain of $\left\{x_{2}=0\right\}$ which contradicts our assumptions.

Moreover, we shall prove that there are no compact curves in $\beta \backslash\left(\gamma_{0}^{+} \cup \gamma_{0}^{-}\right)$ starting at $\gamma_{0}^{+}$and ending at $\gamma_{0}^{-}$. Assume there exists $\tau$ such a curve. As 
there are no compact regions of $M \backslash \beta$, we infer that $\tau$ is the unique curve that starts at $\gamma_{0}^{+}$and ends at $\gamma_{0}^{-}$. Taking into account the above paragraph and the fact that $\Gamma^{+}$and $\Gamma^{-}$are in the same half-slab of $S$ determinated by $\left\{x_{2}=0\right\}$ we conclude that must exist a pair of curves, $\tau_{1}$ and $\tau_{2}$ starting at $\gamma_{0}^{+}$and $\gamma_{0}^{-}$, respectively and diverging to either $E_{1}$ or $E_{2}$. We assert that both curves must diverge to the same end. Indeed, if $\tau_{1}$ and $\tau_{2}$ diverge each one to one different end, then there exists a curve $\tau_{3}$ diverging to both ends. But this curve $\tau_{3}$ intersects $\tau$ transversally in a odd number of points while $X\left(\tau_{3}\right)$ intersects $X(\tau)$ transversally in a even number of points.

Without loss of generality, we can assume that $\tau_{1}$ and $\tau_{2}$ diverge to $E_{1}$. Now, we may consider, taking pieces of $\gamma_{0}^{+}$and $\gamma_{0}^{-}$if necessary, a piecewise analytic curve $\tau^{\prime}$ from $E_{1}$ to $E_{1}$ that encloses a disk $\Omega$ of $M \backslash \beta$. If $X\left(\tau_{1}\right)$ and $X\left(\tau_{2}\right)$ are contained in the same half-strip, the domain $\Omega$ verifies the conditions of statement (i) in Theorem 1 and we obtain a contradiction. Assume that $X\left(\tau_{1}\right)$ and $X\left(\tau_{2}\right)$ are contained in different half-strips. Note that then there is an angle between the curves in $\tau^{\prime}, \Theta$, that goes by $X$ to an angle greater or equal than $2 \Theta$. Using again that $X: M \longrightarrow \mathbb{R}^{3}$ is conformal we get a contradiction.

Consequently, $\beta \backslash\left(\gamma_{0}^{+} \cup \gamma_{0}^{-}\right)$consists of curves starting at $\gamma_{0}^{+} \cup \gamma_{0}^{-}$and diverging to one end and divergent curves. Next we prove that there are no curves diverging to only one end. As before we have that one of these curves would be contained either in $B^{+}$or in $B^{-}$. Otherwise, in each of these cases it is possible to find a connected component, $\Omega$, of $M \backslash \beta$ such that $X(\Omega)$ is contained in a half-slab of $S$ and $X(\partial(\Omega))$ is contained in a half-strip of $\left\{x_{2}=0\right\}$. Consequently, applying Statement (i) in Theorem 1 we obtain that $X(\Omega)$ is a planar domain of $\left\{x_{2}=0\right\}$ which contradicts our assumptions. Furthermore, using again that $\Gamma^{+}$and $\Gamma^{-}$are in one of the half-slabs determinated by $\left\{x_{2}=0\right\}$ we deduce that if there exists a curve that starts at $\gamma_{0}^{ \pm}$and diverge to one end, then there exists a curve that starts at $\gamma_{0}^{ \pm}$and diverge to the other end. All these facts allows us to assert that in $\beta \backslash\left(\gamma_{0}^{+} \cup \gamma_{0}^{-}\right)$either there are no curves starting at $\gamma_{0}^{+} \cup \gamma_{0}^{-}$, or there are a pair starting at $\gamma_{0}^{+}$or $\gamma_{0}^{-}$and diverging to different ends or there are four curves, a pair starting at $\gamma_{0}^{+}$and diverging to different ends and another pair starting at $\gamma_{0}^{-}$and diverging to different ends. Moreover, we may find curves in $\beta \backslash\left(\gamma_{0}^{+} \cup \gamma_{0}^{-}\right)$diverging to the two ends. Note that then the number of curves diverging to $E_{1}$ is the same as the number of curves diverging to $E_{2}$. It is not hard to see, using Statement (i) in Theorem 1, that two consecutive curves diverging to the same end have to be in different half-strips, it is to say, if one is in $B^{+}$the other one is in $B^{-}$and that all divergent curves are disjoint. Assume that there are more than two curves in $\beta$ diverging to $E_{1}$ and consider the compact curves $\sigma^{i}=\sigma_{a}^{i}$, for $i=1,2$ and $a \geq a_{0}$ given in Lemma 1 . 
Now, we analyze each of the possibilities for the boundary separately.

Case 1. Denote $p_{1}^{+}=\gamma_{1}^{+} \cap \sigma^{1}$ and $p_{1}^{-}=\gamma_{1}^{-} \cap \sigma^{1}$. Then, denoting $\mathfrak{p}_{3}$ : $\mathbb{R}^{3} \longrightarrow\left\{x_{3}=0\right\}$ as the orthogonal projection over the plane $\left\{x_{3}=0\right\}$ we deduce that $\mathfrak{p}_{3}\left(X\left(\sigma^{1}\right)\right)$ is a curve in $\left\{x_{3}=0\right\}$ such that $\mid \arg \left(\mathfrak{p}_{3}\left(X\left(p_{1}^{-}\right)\right)\right)$$\arg \left(\mathfrak{p}_{3}\left(X\left(p_{1}^{+}\right)\right)\right) \mid>2 \pi$. Since $X_{3}\left(p_{1}^{+}\right)=X_{3}\left(p_{1}^{-}\right)=\frac{1}{2}$ we infer that $X\left(\sigma^{1}\right)$ has self-intersections, which is contrary to our assumptions. As a consequence, there is at most two curves in $\beta$ diverging to $E_{1}$ and the same for $E_{2}$.

Case 2. Denote $p_{1}^{+}=\gamma_{1}^{+} \cap \sigma^{1}, p_{2}^{-}=\gamma_{2}^{-} \cap \sigma^{1}, p_{2}^{+}=\gamma_{2}^{+} \cap \sigma^{2}$ and $p_{1}^{-}=$ $\gamma_{1}^{-} \cap \sigma^{2}$ and suppose that $\sigma^{1}$ has been parametriced so that it starts at $p_{1}^{+}$ and ends at $p_{2}^{-}$and $\sigma^{2}$ has been parametriced so that it starts at $p_{1}^{-}$and ends at $p_{2}^{+}$. Using the same notation as above we can see that $\mathfrak{p}_{3}\left(X\left(\sigma^{i}\right)\right)$ are curves in $\left\{x_{3}=0\right\}$ satisfying $\left|\arg \left(\mathfrak{p}_{3}\left(X\left(p_{2}^{-}\right)\right)\right)-\arg \left(\mathfrak{p}_{3}\left(X\left(p_{1}^{+}\right)\right)\right)\right|>2 \pi$ and $\left|\arg \left(\mathfrak{p}_{3}\left(X\left(p_{2}^{+}\right)\right)\right)-\arg \left(\mathfrak{p}_{3}\left(X\left(p_{1}^{-}\right)\right)\right)\right|>2 \pi$. Moreover, $\mathfrak{p}_{3}\left(X\left(\sigma^{1}\right)\right)$ and $\mathfrak{p}_{3}\left(X\left(\sigma^{2}\right)\right)$ rotates around $(0,0,0)$ in reverse sense, it is to say, if $\mathfrak{p}_{3}\left(X\left(\sigma^{1}\right)\right)$ rotates clockwise then $\mathfrak{p}_{3}\left(X\left(\sigma^{2}\right)\right)$ rotates counterclockwise, and vice versa. Since $X_{3}\left(p_{i}^{+}\right)=X_{3}\left(p_{i}^{-}\right)$for $i=1,2$ we infer that $X\left(\sigma^{1}\right)$ and $X\left(\sigma^{2}\right)$ intersect each other. This contradicts our assumptions and therefore there is at most two curves diverging to each end in $\beta$.

The same argument used in both cases proves that if $\beta \backslash\left(\gamma_{0}^{+} \cup \gamma_{0}^{-}\right)$consists of two curves, $\tau_{1}$ and $\tau_{2}$, diverging to the two ends such that $X\left(\tau_{1}\right) \subset B^{+}$ and $X\left(\tau_{2}\right) \subset B^{-}$, then the boundaries of the three connected components of $M \backslash \beta$ are $\gamma^{+} \cup \tau_{1}, \tau_{1} \cup \tau_{2}$ and $\gamma^{-} \cup \tau_{2}$.

Taking into account this and the fact that $\Gamma^{+} \cup \Gamma^{-}$is in one of the halfslabs determinated by $\left\{x_{2}=0\right\}$ we have that either $\beta \backslash\left(\gamma_{0}^{+} \cup \gamma_{0}^{-}\right)$is empty or it consists of:

1. Two curves diverging to the two ends (see Figure 4(1)),

2. a curve starting at $\gamma_{0}^{+}$and diverging to $E_{1}$, a curve starting at $\gamma_{0}^{+}$and diverging to $E_{2}$ and a curve diverging to the two ends (see Figure 4(2)),

3 . a curve starting at $\gamma_{0}^{-}$and diverging to $E_{1}$, a curve starting at $\gamma_{0}^{-}$and diverging to $E_{2}$ and a curve diverging to the two ends (see Figure 4(3)),

4. a curve starting at $\gamma_{0}^{+}$and diverging to $E_{1}$, a curve starting at $\gamma_{0}^{+}$ and diverging to $E_{2}$, a curve starting at $\gamma_{0}^{-}$and diverging to $E_{1}$ and a curve starting at $\gamma_{0}^{-}$and diverging to $E_{2}$ (see Figure $4(4)$ ).

Clearly, if $\beta \backslash\left(\gamma_{0}^{+} \cup \gamma_{0}^{-}\right)$is empty we obtain that $X(M)$ is contained in the half-slab $\left\{x_{2} \geq 0\right\}$. Therefore $X(M)$ satisfies the conditions of Statement (ii) in Theorem 1 and so $X(M) \subset \mathcal{E}(\Gamma)$.

Assume that we have one of the other possibilities. Then we shall prove that $X(M) \subset(S \backslash \mathcal{E}(\Gamma)) \cup \Gamma$. Note that it is sufficient to study the connected components of $M \backslash \beta$ whose image is contained in the half-slab $\left\{x_{2} \geq 0\right\}$. Note that these connected components are those whose contains any of the curves $\gamma_{i}^{+}$or $\gamma_{i}^{-}$, for $i=1,2$. At this point, it can be easily check that each 


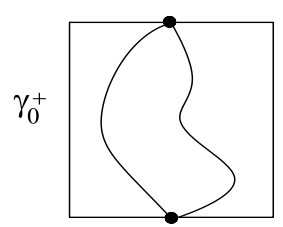

(1)

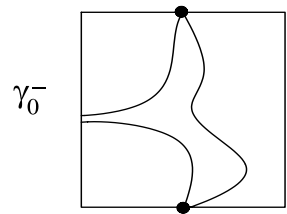

(2)

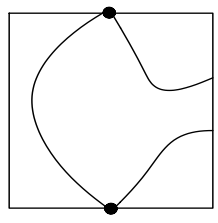

(3)

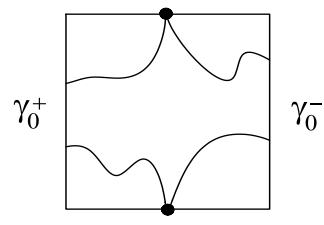

(4)

Figure 4.

of these connected components have the boundary contained in one of the following wedges:

$$
\begin{gathered}
\left(S \backslash \mathcal { E } ( \stackrel { \circ } { \Gamma } ) \cap \left\{\left\{\left(x_{1}, x_{2}, x_{3}\right) \mid x_{1} \geq 0, x_{2} \geq 0, \frac{-1}{2} \leq x_{3} \leq \frac{1}{2}\right\},\right.\right. \\
(S \backslash \mathcal{E}(\Gamma)) \cap\left\{\left(x_{1}, x_{2}, x_{3}\right) \mid x_{1} \leq 0, x_{2} \geq 0, \frac{-1}{2} \leq x_{3} \leq \frac{1}{2}\right\} .
\end{gathered}
$$

Using again Assertion (ii) of Theorem 1, we conclude that the image of these connected components is contained entirely in the correspondent wedge. Summarizing, we have proved that $X(M) \subset(S \backslash \mathcal{E}(\Gamma))$. Now the Proposition is an easy consequence of the maximum principle.

Next, we analyze the case $\theta=\pi, d>0$. Let us define $\Delta=X^{-1}(X(M) \cap$ $\left.\left\{x_{1}=\frac{d}{2}\right\}\right)$. It is well-known that $\Delta$ is a nodal set of an harmonic function and so it is a set of properly immersed analytic lines. Using the maximum principle we obtain that there are no compact connected regions of $M$ bounded by curves in $\Delta$. Then, $\Delta \backslash \gamma_{0}^{+}$consists of a set of divergent curves. Since $\Gamma^{+} \subset\left\{x_{1} \geq \frac{d}{2}\right\}$ and $\Gamma^{-} \subset\left\{x_{1} \leq \frac{d}{2}\right\}$, we infer that if there exist a curve in $\Delta$ starting at $\gamma_{0}^{+}$and diverging to one end, then another curve starting at $\gamma_{0}^{+}$and diverging to the other end must exist. Reasoning as in case $0 \leq \theta<\pi, d=0$ we can see that the image of such a pair of curves is contained in one of the following half-strips:

$$
\begin{aligned}
& C^{+}=\left\{\left(\frac{d}{2}, x_{2}, x_{3}\right) \mid x_{2} \geq 0, \frac{-1}{2} \leq x_{3} \leq \frac{1}{2}\right\}, \\
& C^{-}=\left\{\left(\frac{d}{2}, x_{2}, x_{3}\right) \mid x_{2} \leq 0, \frac{-1}{2} \leq x_{3} \leq \frac{1}{2}\right\},
\end{aligned}
$$

and if two curves in $\Delta$ diverge to the same end there must be one of them with the image contained in $C^{+}$and the other one with the image in $C^{-}$. Therefore, adapting to this situation the argument presented above for the two different possibilities of the boundary, it is not hard to see that there is at most a curve diverging to each end. And then $\Delta \backslash \gamma_{0}^{+}$consists of either a curve diverging to the two ends or a pair of curves starting at $\gamma_{0}^{+}$ and diverging to different ends. Note that in both cases $X(\Delta)$ is contained either in $C^{+}$or $C^{-}$. In order to conclude the proposition it is sufficient 
to apply Statement (ii) in Theorem 1 to each of connected components of $M \backslash \Delta$.

Remark 1. Assume that $T$ is a plane in $\mathbb{R}^{3}$ and that the divergent curves in $X^{-1}(X(M) \cap T)$ verifies that two consecutive divergent curves are in different half-strips of $T$. Then reasoning as in the proof of Proposition 1 we can see that there are at most two curves diverging to each end.

Corollary 1. The boundary of the immersion $X$ is as in Case 1.

Proof. Assuming that $0 \leq \theta<\pi$ and taking into account Proposition 1 we have that either $X(M) \subset \mathcal{E}(M)$ or $X(M) \subset(S \backslash \mathcal{E}(\Gamma)) \cup \Gamma$. Suppose that the boundary behaviour is as in Case 2 and consider the compact curves $\sigma^{i}=\sigma_{a}^{i}, i=1,2$ given in Lemma 1 for some $a \geq a_{0}$. Clearly, $X\left(\sigma^{1}\right)$ starts at $\ell_{1}^{+}$and ends at $\ell_{2}^{-}$and $X\left(\sigma^{2}\right)$ is a curve starting at $\ell_{1}^{-}$and ending at $\ell_{2}^{+}$. Since both curves lie either in $C_{a} \cap \mathcal{E}(\Gamma)$ or in $C_{a} \cap((S \backslash \mathcal{E}(\Gamma)) \cup \Gamma)$, they intersect, which contradicts our assumptions.

Taking the above corollary into account and the fact that $X_{3}$ is a bounded harmonic function one has the following:

Corollary 2. The function $X_{3}: M \longrightarrow \mathbb{R}^{3}$ extends continuously to the ends.

Let us consider $\delta_{t}=X^{-1}\left(X(M) \cap\left\{x_{3}=t\right\}\right)$ for $t \geq-\frac{1}{2}$. Concerning $\delta_{t}$ we can prove:

Corollary 3. The set $\delta_{t}$ is compact and consists of a simple arc, for all $t \in]-\frac{1}{2}, \frac{1}{2}[$. Moreover, the Gauss map $g$ of $X$ omits the points 0 and $\infty$.

Proof. Clearly, from Corollary 2, we deduce that $\delta_{t}$ is compact. Since $\delta_{t}$ is the nodal set of a harmonic function we have that $\delta_{t}$ is a one-dimensional proper real analytic subvariety of $M$. Then, taking into account the maximum principle we deduce that there are no regions in $M$ bounded by curves in $X^{-1}\left(\delta_{t}\right)$. Therefore, $\delta_{t}$ is a regular simple curve in $M$ starting at $\ell_{0}^{+}$and ending at $\ell_{0}^{-}$. Moreover, the theorem of the order of contact (see [13, §437]) gives that there are no points in $M$ with vertical normal vector.

In the case $0 \leq \theta<\pi$ the uniqueness of solutions $X: M \longrightarrow \mathbb{R}^{3}$ of Problem 1 satisfying $X(M) \subset \mathcal{E}(M)$ were completely studied by F.J. López and F. Martín in [9]. Henceforth, in the remainder of the section we assume that $X(M) \subset(S \backslash \mathcal{E}(\Gamma)) \cup \Gamma$. Furthermore, we always assume that $X(M) \subset$ $\left\{x_{2} \leq 0\right\}$ in the case $\theta=\pi$. With this assumptions, we can prove:

Lemma 3. We have the following possibilities for the set $\tau_{0}=X^{-1}(X(M) \cap$ $\left.\left\{x_{1}=0\right\}\right)$ :

i) If $d>0, \tau_{0}$ consists of a curve diverging to both ends, $E_{1}$ and $E_{2}$. 
ii) If $d=0, \tau_{0} \backslash\left(\gamma_{0}^{+} \cup \gamma_{0}^{-}\right)$either consists of a curve diverging to both ends or $\tau_{0} \backslash\left(\gamma_{0}^{+} \cup \gamma_{0}^{-}\right)=\tau_{0}^{1} \cup \tau_{0}^{2}$, where $\tau_{0}^{i}$ are curves starting at $\gamma_{0}^{+}$or $\gamma_{0}^{-}$ and diverging to $E_{i}$, for $i=1,2$.

Proof. Since $\tau_{0}$ is the nodal set of a harmonic function we have that $\tau_{0}$ is a set of properly immersed analytic curves in $M$. Observe that $\tau_{0} \neq \emptyset$. If not, applying Statement (ii) in Theorem 1 we obtain that $X(M)$ are two planar domains. Moreover, by the maximum principle, there are no compact connected regions in $M \backslash \tau_{0}$ bounded by curves in $\tau_{0}$. Clearly, taking into account that $X(M) \cap\left\{x_{1}=0\right\} \subset\left\{\left(x_{1}, x_{2}, x_{3}\right) \mid x_{1}=0, x_{2} \leq 0,-\frac{1}{2} \leq x_{3} \leq\right.$ $\left.\frac{1}{2}\right\}$ and Statement (i) in Theorem 1 we obtain i).

Assume now that $d=0$. In this case we have $\gamma_{0}^{+} \cup \gamma_{0}^{-} \subset \tau_{0}$. Since $\Gamma^{+} \subset\left\{x_{1} \geq 0\right\}$ and $\Gamma^{-} \subset\left\{x_{1} \leq 0\right\}$, we infer that if there exists a curve in $\tau_{0}$ starting at $\gamma_{0}^{ \pm}$and diverging to one end, then there exist another curve which starts at $\gamma_{0}^{ \pm}$and diverges to the other end. Then reasoning as in the above paragraph we obtain ii).

Proposition 2. Counting multiplicities $\sharp\left[N^{-1}\left(-\vec{a}_{2}, \vec{a}_{2}\right)\right] \leq 5$ and

$$
\sharp\left[N^{-1}\left(-\vec{a}_{2}, \vec{a}_{2}\right) \cap(M \backslash \partial(M))\right] \leq 3,
$$

where $N: M \longrightarrow \mathbb{S}^{2}$ is the Gauss map of $X$. Furthermore, if $0<\theta<\pi$ we have

$$
\sharp\left[N^{-1}(-\vec{a}, \vec{a})\right] \leq 5, \forall \vec{a} \in \mathbb{S}^{2} \cap\left\{x_{3}=0\right\} .
$$

Proof. We prove the first assertion in Proposition 2. The second assertion can be proved using similar arguments. Let us consider $\beta_{t}=X^{-1}(X(M) \cap$ $\left.\left\{x_{2}=t\right\}\right)$, for $t \in \mathbb{R}$. Note that $\beta_{t}$ is a set of properly immersed analytic lines, because it is the nodal set of a harmonic function. Hence, using the maximum principle, we infer that there are no compact domains in $M$ bounded by curves in $\beta_{t}$, for all $t \in \mathbb{R}$. Therefore, any two curves in $\beta_{t}$ do not intersect in more than one point. If not, we can find a compact domain of $M \backslash \beta_{t}$ bounded by curves in $\beta_{t}$.

We start with the case $t<0$. Observe that in this case $\beta_{t}$ is a nonempty set of divergent curves, converging to a unique end or to the two ends. If $\beta_{t}=\emptyset$ for some $t<0$ we deduce that $X(M) \subset\left\{x_{2} \geq 0\right\}$ and applying Statement (ii) in Theorem 1 we obtain $X(M) \subset \mathcal{E}(\Gamma)$ which contradicts our assumption.

Let $\widetilde{\alpha}_{1}$ and $\widetilde{\alpha}_{2}$ be a pair of arcs in $\beta_{t}$ diverging to $E_{i}$ such that $\widetilde{\alpha}_{1} \cap \widetilde{\alpha}_{2} \neq \emptyset$. Therefore, there exists $U_{i}$, a neighbourhood of $E_{i}$, verifying that:

- $U_{i} \backslash\left(U_{i} \cap \tau_{0}\right)$ has two connected components, $U_{i}^{+}$and $U_{i}^{-}$, where $\tau_{0}$ was defined in Lemma 3.

- $\widetilde{\alpha}_{1} \cap U_{i} \subset U_{i}^{+}$and $\widetilde{\alpha}_{2} \cap U_{i} \subset U_{i}^{-}$.

If not, one can find a neighbourhood of $E_{i}, U_{i}$, verifying the first condition and such that either $\left(\widetilde{\alpha}_{1} \cup \widetilde{\alpha}_{2}\right) \cap U_{i} \subset U_{i}^{+}$or $\left(\widetilde{\alpha}_{1} \cup \widetilde{\alpha}_{2}\right) \cap U_{i} \subset U_{i}^{-}$. Hence, 
we deduce that $X\left(\left(\widetilde{\alpha}_{1} \cup \widetilde{\alpha}_{2}\right) \cap U_{i}\right), i=1,2$ are contained in a half strip of $\left\{x_{2}=t\right\}$ and so there is a connected component in $M \backslash \beta_{t}, \Omega$, whose boundary is in a half-strip of $\left\{x_{2}=t\right\}$. Therefore, we can apply Statement (i) in Theorem 1 to conclude that $X(\Omega)$ is a planar domain in $\left\{x_{2}=t\right\}$, which is a contradiction.

Let us prove that if $\alpha_{1}$ and $\alpha_{2}$ are two curves in $\beta_{t}$ diverging to $E_{i}$, then they are disjoint. Indeed, if $\alpha_{1}$ and $\alpha_{2}$ intersect then we have four arcs $\left\{\widetilde{\alpha}_{i}\right\}_{i=1}^{4}$ in $\beta_{t}$ diverging to $E_{i}$ and $\widetilde{\alpha}_{j} \cap \widetilde{\alpha}_{l} \neq \emptyset$. But this contradicts the above result.

Assume now that $\alpha_{1}$ is a curve diverging to $E_{i}$ and that $\alpha_{2}$ is a curve diverging to the two ends. If $\alpha_{1}$ and $\alpha_{2}$ intersect each other then we have three $\operatorname{arcs}\left\{\widetilde{\alpha}_{i}\right\}_{i=1}^{3}$ in $\beta_{t}$ diverging to $E_{i}$ and $\widetilde{\alpha}_{j} \cap \widetilde{\alpha}_{l} \neq \emptyset$. But, again this contradicts the above result.

As a consequence, only curves diverging to the two ends can intersect. Now, we shall prove that there are at most two of these curves whose intersection is not empty. Assume there exist $\alpha_{i}$ for $i=1,2,3$ curves in $\beta_{t}$ diverging to the two ends such that $\alpha_{1} \cap \alpha_{i} \neq \emptyset$, for $i=2,3$. Then, by considering apropriate arcs in $\alpha_{i}$, for $i=1,2,3$ and using the assertion proved above about arcs diverging to one end, we can find a connected component of $M \backslash \beta_{t}$ that satisfies the conditions of Statement (i) in Theorem 1 and then $X(\Omega)$ must be a planar domain in $\left\{x_{2}=t\right\}$, which contradicts our assumptions. Moreover, it is clear that if $\alpha_{1}$ and $\alpha_{2}$ are two curves in $\beta_{t}$ diverging to the two ends whose intersection is not empty then $\alpha_{1} \cap \alpha_{2}$ is a unique point.

Lastly, if we have two curves in $\beta_{t}$ diverging to the two ends whose intersection is not empty, then there are no more intersections in $\beta_{t^{\prime}}$ for any $t^{\prime}<0, t^{\prime} \neq t$. If not, using again the above assertion, we deduce that the pair of divergent curves in $\beta_{t}$ intersect the pair of divergent curves in $\beta_{t^{\prime}}$. Since $\beta_{t}$ and $\beta_{t^{\prime}}$ are contained in parallel planes, this is a contradiction.

Now, we tackle the case $t>0$. Observe that this case only has sense if $0 \leq \theta<\pi$ and that $\beta_{t} \cap \partial(M)=p_{1}^{+} \cup p_{2}^{+} \cup p_{1}^{-} \cup p_{2}^{-}$where $p_{i}^{+} \in \gamma_{i}^{+}$ and $p_{i}^{-} \in \gamma_{i}^{-}$, for $i=1,2$. Since $X(M) \subset(S \backslash \mathcal{E}(M)) \cup \Gamma$ we deduce that connected curves in $\beta_{t}$ are contained in a half-strip of $\left\{x_{2}=t\right\}$. Therefore, there are no curves in $\beta_{t}$ diverging to one end. Indeed, we have a connected component of $M \backslash \beta_{t}$ satisfying the conditions in Statement (i) in Theorem 1 and so we get a contradiction. Hence, it is clear that curves in $\beta_{t}$ diverging to two ends are disjoint and moreover a divergent curve starting at $\partial(M)$ and a curve diverging to the two ends can not intersect each other. Then, $\alpha_{1} \cap \alpha_{2} \neq \emptyset$ only in two situations:

i) When $\alpha_{1}$ is a curve starting at $\gamma_{1}^{+}$and diverging to $E_{2}$ and $\alpha_{2}$ is a curve starting at $\gamma_{2}^{+}$and diverging to $E_{1}$, 
ii) when $\alpha_{1}$ is a curve starting at $\gamma_{1}^{-}$and diverging to $E_{2}$ and $\alpha_{2}$ is a curve starting at $\gamma_{2}^{-}$and diverging to $E_{1}$.

Moreover, we observe that if there exist a pair of curves as in i) for $t_{0}>0$, this pair is unique. It is to say, $\left\{x_{2}=t\right\} \cap\left\{x_{1} \geq 0\right\} \cap X\left(N^{-1}\left(\left\{-\vec{a}_{2}, \vec{a}_{2}\right\}\right)\right)=\emptyset$ for $t>0, t \neq t_{0}$. And the same occurs for a pair of curves as in ii). Therefore, we have at most two points in $\delta_{t} \cap N^{-1}\left(\left\{-\vec{a}_{2}, \vec{a}_{2}\right\}\right)$ for $t>0$.

We recall that the set $\beta_{0}=\beta$ was studied in the proof of Proposition 1. Since we are assuming $X(M) \subset(S \backslash \mathcal{E}(\Gamma)) \cup \Gamma$ the possibilities for $\beta_{0}$ are those described in 1,2, 3 and 4 . We also point out that Case 2 is not compatible with Case i) analyzed in the case $t>0$, Case 3 is not compatible with Case ii) analyzed in the case $t>0$ and so Case 4 is not compatible with either i) or ii). Therefore, there exist at most five points of ordinary contact in $M$ and only three of them can lie in $M \backslash \partial(M)$.

Lemma 4. For any $p \in \gamma_{1}^{+} \cup \gamma_{1}^{-} \cup \gamma_{2}^{+} \cup \gamma_{2}^{-}$, counting multiplicities, one has $\sharp\left[g^{-1}(g(p)) \cap \partial(M)\right]<6$.

Proof. Assume $X(p) \in \ell_{1}^{+}$. The proofs of the other possibilities are similar.

Label $\Sigma$ as the tangent plane to $X(M)$ at $X(p)$ and let us consider $\Lambda=$ $X^{-1}(\Sigma \cap X(M))$. Since $\Lambda$ is the nodal set of a harmonic function, then $\Lambda$ is a set of properly immersed analytic curves. Using the interior maximum principle we also deduce that there are no compact simply connected region of $M$ bounded by curves in $\Lambda$.

First, we study the case $0 \leq \theta<\pi$. In this case $\ell_{1}^{+} \subset \Sigma$ and since $X(M) \subset$ $(S \backslash \mathcal{E}(\Gamma)) \cup \Gamma$, it is straightforward to prove that $\Lambda \cap X^{-1}\left(\Gamma-\ell_{1}^{+}\right)=\emptyset$. Hence, taking into account the reasoning at the beginning of the proof and that $M$ is simply connected, we have that if $\lambda$ is a curve in $\Lambda$ starting at $\gamma_{1}^{+}$, then $\lambda$ diverges to an end and when two curves in $\Lambda$ start at $\gamma_{1}^{+}$, they do not intersect.

If $\sharp\left[g^{-1}(g(p)) \cap \partial(M)\right] \geq 6$ then, using once again the theorem of the order of contact (see $[\mathbf{1 3}, \S 437]$ ), there are at least 6 curves in $\Lambda$ starting at $\gamma_{1}^{+}$. Observe that then there exist at least three curves diverging to the same end. Consider this set of diverging curves. If there is a pair of consecutive curves in this set contained in the same half-strip of $\Sigma$ then the connected component between them, that we call $\Omega$, satisfies the conditions of Statement (i) in Theorem 1 and then $X(\Omega)$ must be a planar domain in $\Sigma$, which contradicts our assumptions. On the contrary, if each pair of consecutive curves are in different half-strips, we can use Remark 1 and obtain a new contradiction.

Finally, we analyze the case $\theta=\pi$. Observe that in this case $\Lambda \cap \partial(M)=$ $\gamma_{1}^{+} \cup \gamma_{1}^{-}$. Suppose $\sharp\left[g^{-1}(g(p)) \cap \partial(M)\right] \geq 6$. We note that in this case compact curves starting at $\gamma_{1}^{+}$and ending at $\gamma_{1}^{-}$can appear in $\Lambda$. Otherwise, we can only have one of these curves, because if there exist two or more curves of this type in $\Lambda$ we would get a compact domain, $\Omega$, in $M \backslash \Lambda$ satisfying 
$X(\Omega) \subset \Sigma$, and this is a contradiction. Either there exists a compact curve in $\Lambda$ or $\Lambda$ consists only of divergent curves starting at $\gamma_{1}^{+} \cup \gamma_{1}^{-}$, it is not hard to see, using Statement (i) in Theorem 1, that there are at least a set of three curves (counting the curve $\gamma_{1}^{+}$) diverging to the same end in $\Lambda$ such that two consecutive curves in this set are in different half-strips of $\Sigma$. Then we can conclude as in the former case.

Using the above lemmas we can now prove:

Proposition 3. The map g extends continuously to the ends. In particular, the total curvature of $M$ is finite. Furthermore, the limit tangent plane to $M$ at $E_{i}$ is $\pi_{i}, i=1,2$.

Proof. We shall prove that the map $g$ extends to $E_{1}$. The same argument can be used for $E_{2}$. Taking into account Lemma 4 it is not difficult to prove that the following limits exist:

$$
\lim _{\substack{p \rightarrow E_{1} \\ p \in \gamma^{+}}} g(p), \quad \lim _{\substack{p \rightarrow E_{1} \\ p \in \gamma^{-}}} g(p), \quad i=1,2 .
$$

For the proof of this fact see Claim 3.15 in [9].

Since $M$ is conformally equivalent to a sector $S_{\theta_{1}}=\left\{r \mathrm{e}^{\mathrm{i} \Theta} \mid r>0, \Theta \in\right.$ $\left.\left[0, \theta_{1}\right]\right\}$, a truncated sector $S_{\theta_{1}}(R)=S_{\theta_{1}} \backslash \overline{D(0, R)}$ can be seen as a neighbourhood of $E_{1}$ in $M$. Furthermore, we can assume that $R$ is sufficiently large so that $X\left(r \mathrm{e}^{\mathrm{i} \theta_{1}}\right) \in \ell_{1}^{+}$for $r>R$. According to Schwarz Principle we can consider the reflection respect to $\ell_{1}^{+}$of $X\left(S_{\theta_{1}}(R)\right)$. Taking into account Proposition 2 we deduce that the Gauss map $N$ on the truncated sector $S_{2 \theta_{1}}(R)=\left\{r \mathrm{e}^{\mathrm{i} \Theta} \mid r>0, \Theta \in\left[0,2 \theta_{1}\right]\right\} \backslash \overline{D(0, R)}$, assumes the values $\vec{a}_{2}$ and $-\vec{a}_{2}$ a finite number of times. Then it is possible to choose $R^{\prime}>R$ sufficiently large so that $g$ restricted to $S_{2 \theta_{1}}\left(R^{\prime}\right)$ omits the values -1 and 1 .

At this point, we need the following technical result:

Let $\alpha<\gamma<\beta, S(R)=\left\{r \mathrm{e}^{\mathrm{i} \Theta} \mid r>0, \Theta \in\right] \alpha, \beta[\} \backslash \overline{D(0, R)}$, for $R>0$ and let $f$ be holomorphic in $S(R)$ and for some complex c satisfy $\lim _{r \rightarrow+\infty} f\left(r e^{i \gamma}\right)=c$. Suppose that there are two distinct complex number absent from the range of $f$. Then $\lim _{r \rightarrow+\infty} f\left(r e^{i \Theta}\right)=c$ for every $\left.\Theta \in\right] \alpha, \beta[$.

We refer to reference book [1, pp. 441-445].

This theorem and (1) imply that $g$ extends continuously to $E_{1}$. Finally, since $M \subset S$ we have that the limit tangent plane at $E_{i}$ coincides with $\pi_{i}$, $i=1,2$.

Consider again the compact curves $\sigma_{a}^{i}$ given in Lemma 1 for $i=1,2$ and $a \geq a_{0}$ sufficiently large. Let us denote by $U_{a}^{i}$ the connected component of $M \backslash\left(\sigma_{a}^{1} \cup \sigma_{a}^{2}\right)$ that contains the end $E_{i}, i=1,2$. With this notation we can prove the following useful result: 
Corollary 4. For a sufficiently large, $X\left(U_{a}^{i}\right)$ is a graph over the plane $\pi_{i}$, $i=1,2$.

Proof. Take $a \geq a_{0}$ sufficiently large so that $g\left(U_{a}^{i}\right)$ does not intersect the equator $\{z \in \overline{\mathbb{C}}|| z \mid=1\}$.

Taking into account that $X(M) \subset(S \backslash \mathcal{E}(\Gamma)) \cup \Gamma$ and the definition of $U_{a}^{i}$, it is not hard to see that $\left.\mathfrak{p}_{3}\right|_{X\left(U_{a}^{i}\right)}$ is a local diffeomorphism onto $\Omega_{a}^{i}$, where $\Omega_{a}^{i}$ is the exterior unbounded domain in the plane $\left\{x_{3}=0\right\}$ determined by the curve $\mathfrak{p}_{3}\left(\ell_{i}^{+}\right) \cup \mathfrak{p}_{3}\left(\ell_{i}^{-}\right) \cup \mathfrak{p}_{3}\left(\sigma_{a}^{i}\right)$. As $X$ is proper, the same occurs for the map $\left.\mathfrak{p}_{3} \circ X\right|_{U_{a}^{i}}$. So, $\left.\mathfrak{p}_{3} \circ X\right|_{U_{a}^{i}}$ is a covering map, and taking into account that $\Omega_{a}^{i}$ is simply connected we deduce that $\left.\mathfrak{p}_{3} \circ X\right|_{U_{a}^{i}}$ is one-to-one. This concludes the proof.

2.2. The symmetries of the surface. The method for proving that $\left\{x_{3}=\right.$ $0\}$ is a plane of symmetry of $X(M)$ is based on the well-known Alexandrov's reflection method and consists of a generalization of Schoen's ideas (see [14]) to our particular case of noncompact boundary. For a precise presentation of our result the following notation is required. Recall that $\delta_{t}=X^{-1}(X(M) \cap$ $\left.\left\{x_{3}=t\right\}\right)$. We also denote for $t \geq-1 / 2$ :

$$
\begin{aligned}
& M_{+}(t)=\left\{\left(x_{1}, x_{2}, x_{3}\right) \in X(M) / x_{3} \geq t\right\}, \\
& M_{-}(t)=\left\{\left(x_{1}, x_{2}, x_{3}\right) \in X(M) / x_{3} \leq t\right\} .
\end{aligned}
$$

A thorough reading of the paragraph 3.2 .2 of [9] will convince the readers that, sharpening some arguments, the proof of Theorem 3.24 still works in the case $X(M) \subset(S \backslash \mathcal{E}(\Gamma)) \cup \Gamma$. Then, we have:

Proposition 4. $X(M)$ is symmetric with respect the plane $\left\{x_{3}=0\right\}$. Furthermore, $M_{+}(0) \backslash\left(\ell_{0}^{+} \cup \ell_{0}^{-}\right)$and $M_{-}(0) \backslash\left(\ell_{0}^{+} \cup \ell_{0}^{-}\right)$are graphs over $\left\{x_{3}=0\right\}$.

Now, we recover two consequences of the above proposition that we need in what follows.

Corollary 5. There are only two branch points $R_{0}^{+} \in \gamma^{+}$and $R_{0}^{-} \in \gamma^{-}$of $g$ along $\gamma_{0}^{+} \cup \gamma_{0}^{-}, g$ has multiplicity two at these points and $R_{0}^{+}, R_{0}^{-} \in \delta_{0}$. Furthermore, the set $\mathcal{G}=\{p \in M /|g(p)|=1\}$ consists of $\gamma_{0}^{+} \cup \gamma_{0}^{-} \cup \delta_{0}$.

Corollary 6. The limit normal vectors at the ends are opposite.

In the remainder of the paper and without loss of generality, we assume that

$$
g\left(E_{1}\right)=0, \quad g\left(E_{2}\right)=\infty .
$$

Next we prove that if $d=0$ then $\left\{x_{1}=0\right\}$ is a plane of symmetry of $X(M)$. As in the horizontal symmetry case, the proof is inspired on Alexandrov's reflection method. However, the argument exhibited here is slightly different from classical Alexandrov's technique which uses a family of parallel planes. 
In this case we use the pencil of vertical planes that contains the vertical segment $\ell_{0}=\ell_{0}^{+}=\ell_{0}^{-}$.

For the sake of simplicity, in this paragraph we consider a new set of Cartesian coordinates obtained from the old one by a rotation of $-\frac{\pi}{2}$ around the $x_{3}$-axis. Observe that in the new coordinate the $x_{1}$-axis is the inner bisector of the orthogonal projection of $\ell_{i}^{+}$and $\ell_{i}^{-}$to the plane $\left\{x_{3}=0\right\}$, $i=1,2$, and $\Gamma^{+} \subset\left\{x_{2} \geq 0\right\}$. Moreover, we need to introduce some notation. For $\left.\left.\xi \in\left[0, \pi-\frac{\theta}{2}\right], t \in\right]-\infty, 0\right]$ and a set $A \subset \mathbb{R}^{3}$ we define:

$$
\begin{aligned}
& H_{\xi}=\left\{\left(x_{1}, x_{2}, x_{3}\right) \in \mathbb{R}^{3} \mid \operatorname{Arg}\left(x_{1}+\mathrm{i} x_{2}\right)=\xi\right\}, H_{\xi, t}=H_{\xi}+(t, 0,0), \\
& P_{\xi}=\left\{\left(x_{1}, x_{2}, x_{3}\right) \in \mathbb{R}^{3} \mid \operatorname{Arg}\left(x_{1}+\mathrm{i} x_{2}\right)=\xi-\frac{\pi}{2}\right\}, P_{\xi, t}=P_{\xi}+(t, 0,0), \\
& H_{\xi}^{+}=\left\{\left(x_{1}, x_{2}, x_{3}\right) \in \mathbb{R}^{3} \mid \operatorname{Arg}\left(x_{1}+\mathrm{i} x_{2}\right) \in\left[\xi, \pi-\frac{\theta}{2}\right]\right\}, \\
& H_{\xi}^{-}=\left\{\left(x_{1}, x_{2}, x_{3}\right) \in \mathbb{R}^{3} \mid \operatorname{Arg}\left(x_{1}+\mathrm{i} x_{2}\right) \in\left[-\pi+\frac{\theta}{2}, \xi\right]\right\}, \\
& \delta(\xi)=X(M) \cap H_{\xi}, A_{+}(\xi)=A \cap H_{\xi}^{+}, A_{-}(\xi)=A \cap H_{\xi}^{-}, \\
& \delta(\xi, t)=X(M) \cap H_{\xi, t}, A_{+}(\xi, t)=A \cap\left(H_{\xi}^{+}+(t, 0,0)\right), \\
& A_{-}(\xi, t)=A \cap\left(H_{\xi}^{-}+(t, 0,0)\right),
\end{aligned}
$$

where Arg : $\mathbb{C} \backslash]-\infty, 0] \longrightarrow \mathbb{R}$ denotes the principal argument. Note that $H_{\xi} \perp P_{\xi}$. In addition we label $\mathfrak{s}_{\xi}: \mathbb{R}^{3} \longrightarrow \mathbb{R}^{3}$ and $\mathfrak{s}_{\xi, t}: \mathbb{R}^{3} \longrightarrow$ $\mathbb{R}^{3}$ as the orthogonal symmetries with respect to the planes containing $H_{\xi}$ and $H_{\xi, t}$, respectively. In the same way, we label $\mathfrak{p}_{\xi}: \mathbb{R}^{3} \longrightarrow H_{\xi}$ as the orthogonal projection. With these definitions we denote $A_{+}^{*}(\xi)=\mathfrak{s}_{\xi}\left(A_{+}(\xi)\right)$ and $A_{+}^{*}(\xi, t)=\mathfrak{s}_{\xi, t}\left(A_{+}(\xi, t)\right)$. In particular we denote $M_{+}(\xi)=X(M)_{+}(\xi)$, $M_{+}(\xi, t)=X(M)_{+}(\xi, t), M_{-}(\xi)=X(M)_{-}(\xi), M_{-}(\xi, t)=X(M)_{-}(\xi, t)$ and $\Delta_{\xi}=M_{+}^{*}(\xi) \cap M_{-}(\xi)$. If $\xi \in\left[\frac{\pi}{2}, \pi-\frac{\theta}{2}\right]$ we also consider $\Delta_{\xi, t}=M_{+}^{*}(\xi, t) \cap$ $M_{-}(\xi, t)$.

Since the following argument is valid for all $\xi \in\left[\frac{\pi}{2}, \pi-\frac{\theta}{2}\right]$ and $\left.\left.t \in\right]-\infty, 0\right]$ we omit the parameters $\xi$ and $t$ in the description of the different sets.

With the above notations, it is not difficult to see that

$$
\Delta \cap\left(\Gamma_{+}^{*} \cup \Gamma_{-}\right)=\delta \cap \Gamma
$$

From Proposition 3 and Corollary 4 we can also consider $a_{1}$ sufficiently large so that $a_{1} \geq a_{0}, X\left(U^{i}\right)=X\left(U_{a_{1}}^{i}\right)$ is a graph over the plane $\left\{x_{3}=0\right\}, i=1,2$ and $\left.x_{3}\right|_{X\left(U^{1}\right)}>0$ and $\left.x_{3}\right|_{X\left(U^{2}\right)}<0$, where $a_{0}$ is as in Lemma 1 and $U_{a_{1}}^{i}$ is defined in Subsection 2.1.

Now, we can prove the following assertion:

Claim 1. If $X^{-1}(\Delta \backslash \delta) \subset U^{1} \cup U^{2}$, then $\Delta=\delta$.

The proof of this claim is similar to the proof of Claim 3.19 in [9]. We refer the reader to $[\mathbf{9}]$ for details. 
Now we define the set

$$
\begin{aligned}
\mathcal{I}=\left\{\xi_{1} \in\left[0, \pi-\frac{\theta}{2}\right] \mid\left[M_{+}^{*}(\xi) \cap\left\{x_{3} \geq 0\right\}\right] \succeq\left[M_{-}(\xi) \cap\left\{x_{3} \geq 0\right\}\right],\right. & \\
& \left.\pi-\frac{\theta}{2} \leq \xi \leq \xi_{1}\right\} .
\end{aligned}
$$

Our objective is to prove that $\mathcal{I}=\left[0, \pi-\frac{\theta}{2}\right]$. We divide the proof of this fact into several points:

Claim 2. $\left.] \frac{\pi}{2}, \pi-\frac{\theta}{2}\right] \subset \mathcal{I}$.

If $A, B \subset \mathbb{R}^{3}$, we say that $A \geq_{\xi} B$ provided for every $x \in \mathbb{R}^{3}$ for which $\mathfrak{p}_{\xi}^{-1}(\{x\}) \cap A \neq \emptyset$ and $\mathfrak{p}_{\xi}^{-1}(\{x\}) \cap B \neq \emptyset$, we have that the orthogonal coordinate to $H_{\xi, t}$ of any point in $\mathfrak{p}_{\xi}^{-1}(\{x\}) \cap A$ is equal to or greater than the respective orthogonal coordinate of any point in $\mathfrak{p}_{\xi}^{-1}(\{x\}) \cap B$.

Given $\left.\xi \in] \frac{\pi}{2}, \pi-\frac{\theta}{2}\right]$, we define the set

$$
\left.\mathcal{I}_{\xi}=\{t \in]-\infty, 0\right] \mid M_{+}(\xi, t) \text { is a graph over } H_{\xi, t}
$$

$$
\text { and } \left.M_{+}^{*}(\xi, t) \leq_{\xi} M_{-}(\xi, t)\right\} \text {. }
$$

Our purpose is to show that $\left.\left.\mathcal{I}_{\xi}=\right]-\infty, 0\right]$, for all $\left.\left.\xi \in\right] \frac{\pi}{2}, \pi-\frac{\theta}{2}\right]$. Note that this fact implies $\left.] \frac{\pi}{2}, \pi-\frac{\theta}{2}\right] \subset \mathcal{I}$.

First, we are going to see that $\mathcal{I}_{\xi} \neq \emptyset$. To do this, let $t^{\prime}<0$ such that $X^{-1}\left(M_{+}(\xi, t)\right) \subset U^{1} \cup U^{2}$, where $U^{i}$ are defined as above, $\forall t \leq t^{\prime}$ (observe that $\left.X^{-1}\left(\Delta_{\xi, t}\right) \subset U^{1} \cup U^{2}\right)$. Hence from Claim $1, \Delta_{\xi, t}=\delta_{\xi, t}, t \leq t^{\prime}$.

Then, it is clear that $X^{-1}\left(M_{+}(\xi, t)\right)$ consists of two simply connected components, one of them in $U^{1}$ and the other one in $U^{2}, \forall t \geq t^{\prime}$, and thus $M_{+}(\xi, t)$ is the union of two disjoint graphs $G_{+}^{1}(\xi, t)$ and $G_{+}^{2}(\xi, t)$ over the same simply connected domain $G_{+}(\xi, t)$ in the plane $\left\{x_{3}=0\right\}$. $\emptyset$.

From the definition of $U^{1}$ and $U^{2}$, we have that $\mathfrak{p}_{\xi}\left(G_{+}^{1}\left(t^{\prime}\right)\right) \cap \mathfrak{p}_{\xi}\left(G_{+}^{2}\left(t^{\prime}\right)\right)=$

Let us see that $M_{+}(\xi, t)$ is a graph over the halfplane $H_{\xi, t}, t \leq t^{\prime}$.

First, observe that $\mathfrak{p}_{\xi}$ is injective on $\delta_{\xi, t}, t \leq t^{\prime}$. Indeed, note that $\delta_{\xi, t} \subset$ $H_{\xi, t}$ is a graph over a connected piece of a straight line, and so the $\mathfrak{p}_{\xi}$ is injective.

Moreover, a similar argument gives that the set $P_{\xi, s} \cap M_{+}\left(\xi, t^{\prime}\right)$ is a connected curve, for sufficiently large $s$. Furthermore, the function $x_{3}$ is monotone over $P_{\xi, s} \cap M_{+}\left(\xi, t^{\prime}\right)$. Otherwise, there would exist some points in $M_{+}\left(\xi, t^{\prime}\right)$ whose normal vector lie in $\left\{x_{1}=0\right\} \cap \mathbb{S}^{2}$. Thus, we could take $t \leq t^{\prime}$ in such a way that $\delta_{t}$ contains a point with normal vector in $P_{\xi} \cap \mathbb{S}^{2}$. Hence, from the theorem of the order of contact (see [13, §437]) and taking into account that $X\left(U^{i}\right)$ are graphs over the plane $x_{3}=0, i=1,2$, we deduce that $\Delta_{\xi, t}-\delta_{\xi, t} \neq \emptyset$, which is contrary to Claim 1 . 
This proves that $M_{+}\left(\xi, t^{\prime}\right)$ is a graph over the plane $H_{\xi}$, and so the same holds for $M_{+}(\xi, t), t \leq t^{\prime}$.

Taking into account that $\Delta_{\xi, t}=\delta_{\xi, t}$, for $t \leq t^{\prime}$ and $X(M) \in(S \backslash \mathcal{E}(M)) \cup \Gamma$, we deduce that $M_{+}^{*}(\xi, t) \leq_{\xi} M_{-}(\xi, t), t \leq t^{\prime}$. Thus, $\left.]-\infty, t^{\prime}\right] \subset \mathcal{I}_{\xi}$.

It is obvious that $\mathcal{I}_{\xi}$ is closed in ] $\left.-\infty, 0\right]$.

Next, we shall see that $0=\operatorname{Maximum}\left(\mathcal{I}_{\xi}\right)$. We proceed by contradiction. Assume $t_{0}=\operatorname{Maximum}\left(\mathcal{I}_{\xi}\right)<0$. Let $K=X\left(M \backslash\left(U^{1} \cup U^{2}\right)\right)$. Since $t_{0} \in \mathcal{I}_{\xi}$, $K_{+}\left(\xi, t_{0}\right)$ is a graph over the plane $H_{\xi}$. Using the interior maximum principle and the maximum principle at the boundary, it is not hard to see that there exists $\varepsilon>0$ such that $K_{+}(\xi, t)$ is a graph over $H_{\xi}$ and $\Delta_{\xi, t} \cap K=\delta_{\xi, t} \cap K$ for $\left.t \in] t_{0}, t_{0}+\varepsilon\right]$ (for details, see Claim 3.21 in [9]). Hence, using Claim 1, we deduce that $\Delta_{\xi, t}=\delta_{\xi, t}$. However, the maximality of $t_{0}$ leads us to $\Delta_{\xi, t} \backslash \delta_{\xi, t} \neq \emptyset$, which is absurd. This finishes the proof of the claim.

Claim 3. The set $\mathcal{I}$ is closed in $\left[0, \pi-\frac{\theta}{2}\right]$ and its minimum is 0 .

Obviously, $\mathcal{I}$ is closed. To prove $\operatorname{Minimum}(\mathcal{I})=0$ we proceed by contradiction. Let us assume that $\operatorname{Minimum}(\mathcal{I})=\xi_{0}>0$. As in the preceding claim, we consider $K=X\left(M \backslash\left(U^{1} \cap U^{2}\right)\right)$. Taking into account that $\xi_{0} \in \mathcal{I}$ we have

$$
\left[M_{+}^{*}\left(\xi_{0}\right) \cap\left\{x_{3} \geq 0\right\}\right] \succeq\left[M_{-}\left(\xi_{0}\right) \cap\left\{x_{3} \geq 0\right\}\right] .
$$

Hence, since $K_{+}\left(\xi_{0}\right)$ is compact, there exists $\varepsilon_{0}>0$ sufficiently small so that $\left[K_{+}^{*}\left(\xi_{0}-\varepsilon\right) \cap\left\{x_{3} \geq 0\right\}\right] \succeq\left[K_{-}\left(\xi_{0}-\varepsilon\right) \cap\left\{x_{3} \geq 0\right\}\right]$, for all $0 \leq \varepsilon \leq \varepsilon_{0}$. Otherwise, we could find sequences $\left\{\xi_{n}\right\} \nearrow \xi_{0}$, with $\xi_{n} \in\left[0, \xi_{0}\left[\right.\right.$, and $\left\{x_{n}\right\}$, $\left\{y_{n}\right\}$ in $K$, fulfilling the following conditions:

i) $x_{n} \in K_{+}\left(\xi_{n}\right), y_{n} \in K_{-}\left(\xi_{n}\right)$ and $\mathfrak{s}_{\xi_{n}}\left(x_{n}\right)=y_{n}, \forall n \in \mathbb{N}$.

ii) $\left\{x_{n}\right\} \rightarrow x \in K_{+}\left(\xi_{0}\right),\left\{y_{n}\right\} \rightarrow y \in K_{-}\left(\xi_{0}\right)$.

From i) and ii) we deduce that $\mathfrak{s}_{\xi_{0}}(x)=y$. On the other hand, (3) implies that any point lying in $\Delta_{\xi_{0}} \backslash \delta_{\xi_{0}}$ is an interior point of contact between $M_{+}^{*}\left(\xi_{0}\right)$ and $M_{-}\left(\xi_{0}\right)$. Assume $\Delta_{\xi_{0}} \backslash \delta_{\xi_{0}} \neq \emptyset$. Then, making use of the interior maximum principle we deduce $M_{+}^{*}\left(\xi_{0}\right)=M_{-}\left(\xi_{0}\right)$, which is absurd because, since $\xi_{0}>0, \Gamma$ is not symmetric with respect to the plane $H_{\xi_{0}}$.

Therefore $x=y \in K \cap \delta_{\xi_{0}}$. Hence, taking into account i) and ii), we have that $N\left(X^{-1}(x)\right)=\mathfrak{s}_{\xi_{0}}\left(N\left(X^{-1}(x)\right)\right)$ and so $N\left(X^{-1}(x)\right)$ is parallel to $H_{\xi_{0}}$. Therefore, by the theorem of the order of contact (see [13, §437]) we have that $x \in K \cap\left(\delta_{\xi_{0}} \backslash\left(\delta_{\xi_{0}} \cap \ell_{0}\right)\right)$ and $x \notin\left\{q_{1}^{+}=q_{1}^{-}, q_{2}^{+}=q_{2}^{-}\right\}$. From this fact and taking into account (4), the maximum principle at the boundary can be applied to a neighbourhood of the point $x$. We get $M_{+}^{*}\left(\xi_{0}\right)=M_{-}\left(\xi_{0}\right)$, which is as above a contradiction.

By the preceding reasoning, we have $\Delta_{\xi} \backslash \delta_{\xi} \subset X\left(U^{1} \cup U^{2}\right)$ for $\xi \geq \xi_{0}-\varepsilon_{0}$. From Claim 1 we conclude that $\Delta_{\xi}=\delta_{\xi}$ for $\xi \geq \xi_{0}-\varepsilon_{0}$. Clearly this implies $\left[M_{+}^{*}(\xi) \cap\left\{x_{3} \geq 0\right\}\right] \succeq\left[M_{-}(\xi) \cap\left\{x_{3} \geq 0\right\}\right]$ for $\xi \geq \xi_{0}-\varepsilon_{0}$ and so $\xi_{0}-\varepsilon_{0} \in \mathcal{I}$, which contradicts that $\xi_{0}$ is a minimum. 
Summarizing we have $\left[M_{+}^{*}(0) \cap\left\{x_{3} \geq 0\right\}\right] \succeq\left[M_{-}(0) \cap\left\{x_{3} \geq 0\right\}\right]$. We can repeat the above argument starting from $\Gamma^{-}$instead of $\Gamma^{+}$and obtain $\left[M_{-}^{*}(0) \cap\left\{x_{3} \geq 0\right\}\right] \succeq\left[M_{+}(0) \cap\left\{x_{3} \geq 0\right\}\right]$ and so $X(M) \cap\left\{x_{3} \geq 0\right\}$ is symmetric with respect to the plane $\left\{x_{2}=0\right\}$. Finally, by the horizontal symmetry mentioned in 2.2 we have that $X(M)$ is symmetric with respect to the plane $\left\{x_{2}=0\right\}$.

\subsection{Determination of conformal structure and Weierstrass data} of $\boldsymbol{M}$. This subsection is devoted to determining the Weierstrass data associated to the minimal immersion $X: M \longrightarrow \mathbb{R}^{3}$. We define $n=\frac{2 \pi}{3 \pi-\theta}$. Observe that $n \in\left[\frac{2}{3}, 1\right]$. As $M$ is simply-connected, the map $(-\mathrm{i} g)^{\frac{n}{2}}$ has a well-defined branch on $M$. Let $f$ be the branch of $(-\mathrm{i} g)^{\frac{n}{2}}$ such that $\operatorname{Arg}(f(p))=0$, whenever $-\mathrm{i} g(p) \in \mathbb{R}^{+}$.

As before, $Q_{j}^{+}=\gamma_{0}^{+} \cap \gamma_{j}^{+}, Q_{j}^{-}=\gamma_{0}^{-} \cap \gamma_{j}^{-}, j=1,2$. Observe that $f\left(Q_{j}^{+}\right)=\mathrm{i}$ and $f\left(Q_{j}^{-}\right)=-\mathrm{i}, j=1,2$. Moreover, since $X(M) \subset(S \backslash \mathcal{E}(\Gamma)) \cup \Gamma$ it is easy to see that $f(p) \neq f\left(Q_{j}^{ \pm}\right)$for all $p \in \gamma_{j}^{ \pm}, j=1,2$. Then, taking into account that $g\left(E_{1}\right)=0$ and $g\left(E_{2}\right)=\infty$, one has:

$$
\begin{gathered}
f\left(\gamma_{1}^{-}\right)=s_{1}^{-}=\left\{\lambda \mathrm{i} \mid \lambda \in\left[-1,0[\}, \quad f\left(\gamma_{2}^{-}\right)=s_{2}^{-}=\{\lambda \mathrm{i} \mid \lambda \in]-\infty,-1\right]\right\}, \\
\left.\left.f\left(\gamma_{1}^{+}\right)=s_{1}^{+}=\{\lambda \mathrm{i} \mid \lambda \in] 0,1\right]\right\}, \quad f\left(\gamma_{2}^{+}\right)=s_{2}^{+}=\{\lambda \mathrm{i} \mid \lambda \in[1, \infty[\} .
\end{gathered}
$$

Using Corollary 5 , we deduce that $\left.f\right|_{\left[Q_{j}^{ \pm}, R_{0}^{ \pm}\right]}$are injective for $j=1,2$. Hence, if we write $f\left(R_{0}^{+}\right)=\mathrm{e}^{\mathrm{i} \frac{t_{0}}{2}}$ and $f\left(R_{0}^{-}\right)=\mathrm{e}^{\mathrm{i} \frac{t_{1}}{2}}$, it is not hard to check that

$$
f\left(\gamma_{0}^{-}\right)=s_{0}^{-}=\left\{\mathrm{e}^{\mathrm{i} \frac{t}{2}} \mid t \in\left[-\pi, t_{1}\right]\right\}, \quad f\left(\gamma_{0}^{+}\right)=s_{0}^{+}=\left\{\mathrm{e}^{\mathrm{i} \frac{t}{2}} \mid t \in\left[t_{0}, \pi\right]\right\} .
$$

Using again that $X(M) \subset(S \backslash \mathcal{E}(\Gamma)) \cup \Gamma$, the fact that there are at most two points on $\gamma_{0}^{ \pm}$where the Gauss map achieves the values $\pm \vec{a}_{2}$ (see the proof of Proposition 1) and Corollary 5, one deduces that $-\pi<t_{1}<0<t_{0}<\pi$.

Let $\Lambda$ denote the connected component of $\mathbb{C} \backslash(f(\partial(M)) \cup\{0\})$ containing the point $\{1\}$ (see Figure 5). We have the following result:

Lemma 5. The map $f: M \rightarrow \mathbb{C}$ fulfills that:

(i) $f(M \backslash \partial(M))=\Lambda$, and

(ii) $\left.f\right|_{M \backslash \partial(M)}: M \backslash \partial M \rightarrow \Lambda$ is a biholomorphism.

Proof. In order to prove (i) we note that $f$ is holomorphic and nonconstant, and so $f(M \backslash \partial(M))$ is an open subset of $\mathbb{C}^{*}$ (note that no points in $M$ have vertical normal vector). On the other hand, taking into account that $\bar{M}=M \cup\left\{E_{1}, E_{2}\right\}$ is compact, $f(\bar{M})$ is a closed subset of $\overline{\mathbb{C}}$. Therefore the set $W=f(M \backslash \partial(M)) \cap(\mathbb{C} \backslash(f(\partial(M)) \cup\{0\}))=f(\bar{M}) \cap(\mathbb{C} \backslash(f(\partial(M)) \cup$ $\{0\}))$ is a closed subset of $\mathbb{C} \backslash(f(\partial(M)) \cup\{0\})$. Then, either $W=\mathbb{C} \backslash$ $(f(\partial(M)) \cup\{0\})$ or $W$ is a connected component of $\mathbb{C} \backslash(f(\partial(M)) \cup\{0\})$. 
To see that the first possibility does not occur we proceed by contradiction. Assume $W=\mathbb{C} \backslash(f(\partial(M)) \cup\{0\})$. Observe that $f(p) \in \mathbb{S}^{1}$ if and only if $g(p) \in \mathbb{S}^{1}$. Hence, taking into account Corollary 5 and (5) we infer that $\left\{\mathrm{e}^{\mathrm{i} \frac{t}{2}}, t \in\left[t_{1}, t_{0}\right] \cup\left[\frac{\pi}{2}, \frac{3 \pi}{2}\right]\right\} \subset f\left(\delta_{0}\right)$. Moreover, since there are no ramification points in $\delta_{0} \backslash\left\{R_{0}^{+}, R_{0}^{-}\right\}$, we deduce that $\operatorname{Arg}(f)$ is a monotone function in $\delta_{0}$ and $f\left(\delta_{0}\right)=\mathbb{S}^{1}$. Since $\left\{1, \mathrm{e}^{\frac{1}{2} n \pi \mathrm{i}}, \mathrm{e}^{n \pi \mathrm{i}}, \mathrm{e}^{\frac{3}{2} n \pi \mathrm{i}}\right\} \subset \mathbb{S}^{1}$, we have that there are at least four points in $\delta_{0} \cap N^{-1}\left(\left\{\vec{a}_{2},-\vec{a}_{2}\right\}\right)$. But it is contrary to Proposition 2. Since $f\left(\gamma_{0}^{+}\right)$and $f\left(\gamma_{0}^{-}\right)$lie in the boundary of $W$, we easily obtain that $W=\Lambda$ and so $f(M \backslash \partial(M))=\Lambda$.

The same argument presented above gives us that $f\left(\delta_{0}\right)=\left\{\mathrm{e}^{\mathrm{i} \frac{t}{2}} \mid t \in\right.$ $\left.\left[t_{1}, t_{0}\right]\right\}$ and $\left.f\right|_{\delta_{0}}: \delta_{0} \longrightarrow\left\{\mathrm{e}^{\mathrm{i} \frac{t}{2}} \mid t \in\left[t_{1}, t_{0}\right]\right\}$ is a one-to-one function.

To finish the proof, we define $\gamma=\partial(\bar{M})$. Since $\bar{M}$ is conformally a closed disk with piecewise analytic boundary, then $\gamma$ is a piecewise analytic curve homeomorphic to $\mathbb{S}^{1}$. Note that since $\left.g\right|_{M}$ has no poles and $g$ extends continuously to $\bar{M}$, we can assert the same for $f$. Then, we know that for any $w \in \Lambda=f(M \backslash \partial(M)), \sharp\left(f^{-1}(w)\right)=\frac{1}{2 \pi \mathrm{i}} \int_{\gamma} \frac{d f}{f-w} \in \mathbb{Z}$. Thus, if we define $h: \Lambda \longrightarrow \mathbb{Z}$ by $h(w)=\sharp\left(f^{-1}(w)\right)$, the function $h$ is continuous on $\Lambda$, and so it is constant. From the above arguments we have that $h(w)=1$, $\forall w \in f\left(\delta_{0} \backslash\left\{R_{0}^{+}, R_{0}^{-}\right\}\right)$and this concludes the proof.

Let $\mathcal{N}_{t_{0}}^{t_{1}}$ be the following four-punctured torus:

$$
\mathcal{N}_{t_{0}}^{t_{1}}=\left\{(u, v) \in \mathbb{C}^{*} \times \mathbb{C} \mid v^{2}=\left(u-\mathrm{e}^{\mathrm{i} \frac{t_{0}}{2}}\right)\left(u+\mathrm{e}^{-\mathrm{i} \frac{t_{0}}{2}}\right)\left(u-\mathrm{e}^{\mathrm{i} \frac{t_{1}}{2}}\right)\left(u+\mathrm{e}^{-\mathrm{i} \frac{t_{1}}{2}}\right)\right\} .
$$

Consider $\mathcal{U}_{t_{0}}^{t_{1}} \subset \mathcal{N}_{t_{0}}^{t_{1}}$ as the connected component of $u^{-1}(\mathbb{C} \backslash f(\partial(M)))$ containing the point $P_{0}=\left(1,2 \sqrt{-\sin \left(\frac{t_{0}}{2}\right) \sin \left(\frac{t_{1}}{2}\right)}\right)$. Define $\mathcal{N}^{\prime}=\overline{\mathcal{U}_{t_{0}}^{t_{1}}}$. At this point we prove the following proposition:

Proposition 5. $M$ is biholomorphic to $\mathcal{N}^{\prime}$. Furthermore, the Weierstrass data are given on $\mathcal{N}^{\prime}$ by

$$
g(u)=\mathrm{i} u^{\frac{2}{n}}, \quad \Phi_{3}=\lambda \frac{d u}{v},
$$

where $\lambda \in \mathbb{R}^{+}$and we choose the branch of $u^{\frac{2}{n}}$ satisfying $1^{\frac{2}{n}}=1$.

Proof. Consider $f: M \backslash \partial(M) \longrightarrow \Lambda$, the biholomorphism defined in Lemma 5 . Observe that the $u$-projection is a biholomorphism from $\mathcal{N}^{\prime} \backslash \partial\left(\mathcal{N}^{\prime}\right)$ onto $\Lambda$ and so $F=f^{-1} \circ u: \mathcal{N}^{\prime} \backslash \partial\left(\mathcal{N}^{\prime}\right) \longrightarrow M \backslash \partial(M)$ is a biholomorphism. Since $\mathcal{N}^{\prime} \backslash \partial\left(\mathcal{N}^{\prime}\right)$ and $M \backslash \partial(M)$ are conformally equivalent to Jordan regions in $\mathbb{C}$, a well-known result of complex analysis asserts that $F$ can be extended to a biholomorphism from $\mathcal{N}^{\prime}$ onto $M$. For the sake of simplicity, in what follows we identify $M$ with $\mathcal{N}^{\prime}$.

Now, we consider the holomorphic function $\omega=\frac{\Phi_{3} v}{d u}$ on $\mathcal{N}^{\prime}$. Let $p_{0} \in$ $s_{0}^{+} \cup s_{0}^{-}$and $\left\{p_{0}^{1}, p_{0}^{2}\right\}=u^{-1}\left(p_{0}\right)$. Since the surface is symmetric with respect 
to the plane $\left\{x_{3}=0\right\}$ (see 2.2), we have that $\Phi_{3}\left(p_{0}^{1}\right)=-\Phi_{3}\left(p_{0}^{2}\right)$. But this is also the behaviour of $v$. Consequently, $\widetilde{\omega}=\omega \circ u^{-1}$ is a holomorphic function in $\Lambda$ that can be extended to $s_{0}^{+} \cup s_{0}^{-}$.

As $\Phi_{3}$ and $v$ extend to the punctures in a natural way, $\widetilde{\omega}$ can be seen as a holomorphic function on $\{z \in \mathbb{C} \mid \operatorname{Re}(z) \geq 0\}$. Furthermore, $\widetilde{\omega}$ is real on $\{z \in \mathbb{C} \mid \operatorname{Re}(z)=0\}$ and so, using the Schwarz Principle, $\widetilde{\omega}$ extends to the whole $\mathbb{C}$. Then, $\widetilde{\omega}$ is a holomorphic function on $\mathbb{C}$ without zeroes or poles and therefore $\widetilde{\omega}=\lambda \in \mathbb{C}^{*}$. Finally, using again that $\widetilde{\omega}$ is real on $\{z \in \mathbb{C} \mid \operatorname{Re}(z)=0\}$ we deduce $\lambda \in \mathbb{R}^{*}$. Observe that, up to a rigid motion, we can assume that $\lambda \in \mathbb{R}^{+}$.

As we announced, we now prove that $X(M)$ also inherits the vertical symmetry of its boundary when $d>0$.

Proposition 6. In the above setting, $t_{1}=-t_{0}$.

Proof. First of all, observe that the result about the vertical symmetry proved in Paragraph 2.2 implies $t_{1}=-t_{0}$ if $d=0$. We shall see that this fact suffices to prove the general case.

Since $X: M \longrightarrow \mathbb{R}^{3}$ is a solution of Problem 1 we have that $X_{1}\left(R_{0}^{-}\right)=-\frac{d}{2}$ and $X_{1}\left(R_{0}^{+}\right)=\frac{d}{2}$. Thus

$$
d=X_{1}\left(R_{0}^{+}\right)-X_{1}\left(R_{0}^{-}\right)=\operatorname{Re} \int_{\widehat{\delta}} \Phi_{1},
$$

where $\widehat{\delta}$ is the lift to $M$ of the curve $\mathrm{e}^{\mathrm{i} \frac{t}{2}}, t_{1} \leq t \leq t_{0}$, in the $u$-plane. Taking into account the expressions for $g$ and $\Phi_{3}$ given in Proposition 5 , it is not difficult to obtain that

$$
d=\frac{\lambda}{4} f_{1}\left(t_{0}, t_{1}\right)=\frac{\lambda}{4} \int_{t_{1}}^{t_{0}} \frac{\cos \left(\frac{t}{n}\right)}{v\left(t, t_{0}, t_{1}\right)} d t
$$

where $v\left(t, t_{0}, t_{1}\right)=\sqrt{\left(\sin \left(\frac{t_{0}}{2}\right)-\sin \left(\frac{t}{2}\right)\right)\left(\sin \left(\frac{t}{2}\right)-\sin \left(\frac{t_{1}}{2}\right)\right)}$. Furthermore, we have that $X_{2}\left(R_{0}^{+}\right)=X_{2}\left(R_{0}^{-}\right)$. Thus

$$
X_{2}\left(R_{0}^{+}\right)-X_{2}\left(R_{0}^{-}\right)=\operatorname{Re} \int_{\widehat{\delta}} \Phi_{2}=0 .
$$

A direct computation using again the expressions of $g$ and $\Phi_{3}$ given in Proposition 5 gives

$$
\operatorname{Re} \int_{\widehat{\delta}} \Phi_{2}=\frac{\lambda}{4} f_{2}\left(t_{0}, t_{1}\right)=\frac{\lambda}{4} \int_{t_{1}}^{t_{0}} \frac{\sin \left(\frac{t}{n}\right)}{v\left(t, t_{0}, t_{1}\right)} .
$$

From the definitions of the functions $f_{1}$ and $f_{2}$ we have

$$
f_{1}\left(t_{0}, t_{1}\right)=f_{1}\left(-t_{1},-t_{0}\right), \quad f_{2}\left(t_{0}, t_{1}\right)=-f_{2}\left(-t_{1},-t_{0}\right),
$$

for all $\left(t_{0}, t_{1}\right) \in[0, \pi[\times]-\pi, 0]$. Observe that if $X: M \longrightarrow \mathbb{R}^{3}$ is the solution of Problem 1 given in Proposition 5 , then $\left(t_{0}, t_{1}\right)$ must satisfy $f_{1}\left(t_{0}, t_{1}\right) \geq$ 
0 and $f_{2}\left(t_{0}, t_{1}\right)=0$. From the properties of $f_{1}$ and $f_{2}$ given in (6), it suffices to study the zeros of the functions $f_{1}$ and $f_{2}$ in the triangle given by $T=\left\{\left(t_{0}, t_{1}\right) \in[0, \pi[\times]-\pi, 0] \mid t_{0} \leq-t_{1}\right\}$.

Let us denote by $L_{1}=\{0\} \times[-\pi, 0], L_{2}=\left\{\left(t_{0}, t_{1}\right) \in[0, \pi] \times[-\pi, 0] \mid t_{0}=\right.$ $\left.-t_{1}\right\}$ and $L_{3}=[0, \pi] \times\{-\pi\}$ the sides of the triangle $T$. We also define the sets $C_{1}=\left\{\left(t_{0}, t_{1}\right) \in T \mid f_{1}\left(t_{0}, t_{1}\right)=0\right\}, C_{2}=\left\{\left(t_{0}, t_{1}\right) \in T \mid f_{2}\left(t_{0}, t_{1}\right)=0\right\}$. It is clear from (6) that $L_{2} \subset C_{2}$. Furthermore, by the vertical symmetry proved in Paragraph 2.2 we deduce that $C_{1}$ can only intersect $C_{2}$ in points of $L_{2}$. For the sake of clarity, we divide the rest of the proof in several steps.

Step 1. The objective of this step is to show that $C_{i}$ is a set of analytic curves in $T$, for $i=1,2$.

Consider the meromorphic 1-form given by $\Phi=\frac{u^{\frac{2}{n}}}{v} d u$. Note that $-f_{2}$ and $f_{1}$ are the real and imaginary part of $4 \int_{\widehat{\delta}} \Phi$. By deriving, one has

$$
\mathcal{L}(\Phi)=\frac{\partial^{2} \Phi}{\partial t_{0}^{2}}+\frac{\partial^{2} \Phi}{\partial t_{1}^{2}}+a_{1}^{1} \frac{\partial \Phi}{\partial t_{0}}+a_{1}^{2} \frac{\partial \Phi}{\partial t_{1}}+a_{0}^{0} \Phi=d(F),
$$

where

$$
\begin{aligned}
& a_{1}^{1}=\frac{\cos \left(t_{0}\right)+\sin \left(\frac{t_{0}}{2}\right) \sin \left(\frac{t_{1}}{2}\right)}{2 \cos \left(\frac{t_{0}}{2}\right)\left(\sin \left(\frac{t_{0}}{2}\right)-\sin \left(\frac{t_{1}}{2}\right)\right)}, \\
& a_{1}^{2}=-\frac{\cos \left(t_{1}\right)+\sin \left(\frac{t_{0}}{2}\right) \sin \left(\frac{t_{1}}{2}\right)}{2 \cos \left(\frac{t_{1}}{2}\right)\left(\sin \left(\frac{t_{0}}{2}\right)-\sin \left(\frac{t_{1}}{2}\right)\right)}, \\
& a_{0}^{0}=\frac{4-n^{2}}{4 n^{2}}
\end{aligned}
$$

and $F$ is the following meromorphic function:

$$
F=\frac{u^{\frac{2}{n}+1}\left(a u^{4}+b u^{3}+c u^{2}+d u+e\right)}{4 n v^{3}},
$$

where $a=2+n, b=-\mathrm{i}(4+n)\left(\sin \left(\frac{t_{0}}{2}\right)+\sin \left(\frac{t_{1}}{2}\right)\right), c=-4\left(1+2 \sin \left(\frac{t_{0}}{2}\right) \sin \left(\frac{t_{1}}{2}\right)\right)$, $d=-\mathrm{i}(-4+n)\left(\sin \left(\frac{t_{0}}{2}\right)+\sin \left(\frac{t_{1}}{2}\right)\right)$ and $e=2-n$.

Integrating by parts in (7), we have that $f_{1}$ and $f_{2}$ are zeroes of the second order elliptic operator given by $\mathcal{L}$. As $C_{1}$ and $C_{2}$ are the nodal sets of $f_{1}$ and $f_{2}$, respectively, we can assert (see [2]) that $C_{i}$ is a set of regular curves and the critical points on the nodal lines are isolated. Furthermore, when the nodal lines meet, they form an equiangular system. Moreover, by the Maximum Principle for elliptic operators, $C_{i}$ cannot contain closed curves.

Step 2. The purpose of the present step is to study the behaviour of the curves in $C_{1}$ at the boundary of the triangle $T$. We shall see that $C_{1} \cap L_{1}=$ $\left(0, \widetilde{t}_{1}\right), C_{1} \cap L_{2}=\left(\widetilde{t}_{0},-\widetilde{t}_{0}\right)$ and that there are no curves in $C_{1}$ approaching $L_{3}$. 
First, we shall prove that $\frac{\partial f_{1}}{\partial t_{1}}\left(t_{0}, t_{1}\right)>0$, for all $\left(t_{0}, t_{1}\right) \in T$. It is not difficult to see that

$$
\begin{aligned}
\frac{\partial}{\partial t_{1}}\left(\frac{\cos \left(\frac{t}{n}\right)}{v\left(t, t_{0}, t_{1}\right)}\right)= & \frac{\cos \left(\frac{t}{n}\right) \cos \left(\frac{t_{1}}{2}\right)-\cos \left(\frac{t}{2}\right) \cos \left(\frac{t_{1}}{n}\right)}{4 v\left(t, t_{0}, t_{1}\right)\left(\sin \left(\frac{t}{2}\right)-\sin \left(\frac{t_{1}}{2}\right)\right)} \\
& -\frac{\cos \left(\frac{t_{1}}{n}\right)}{\sin \left(\frac{t_{0}}{2}\right)-\sin \left(\frac{t_{1}}{2}\right)} d\left(\frac{\sin \left(\frac{t_{0}}{2}\right)-\sin \left(\frac{t}{2}\right)}{v\left(t, t_{0}, t_{1}\right)}\right) .
\end{aligned}
$$

Thus integrating by parts in the above equality we obtain

$$
\frac{\partial f_{1}}{\partial t_{1}}\left(t_{0}, t_{1}\right)=\int_{t_{1}}^{t_{0}} \frac{\cos \left(\frac{t}{n}\right) \cos \left(\frac{t_{1}}{2}\right)-\cos \left(\frac{t}{2}\right) \cos \left(\frac{t_{1}}{n}\right)}{4 v\left(t, t_{0}, t_{1}\right)\left(\sin \left(\frac{t}{2}\right)-\sin \left(\frac{t_{1}}{2}\right)\right)} .
$$

In order to prove $\frac{\partial f_{1}}{\partial t_{1}}\left(t_{0}, t_{1}\right)>0$ we shall see that the function

$$
h(t, n)=\cos \left(\frac{t}{n}\right) \cos \left(\frac{t_{1}}{2}\right)-\cos \left(\frac{t}{2}\right) \cos \left(\frac{t_{1}}{n}\right) \geq 0,
$$

for $t \in\left[t_{1}, t_{0}\right]$. Since $h(-t, n)=h(t, n)$ and $t_{0} \leq-t_{1}$, it suffices to prove that $h(t, n) \geq 0$ for $t \in\left[t_{1}, 0\right]$. Moreover, taking into account $h\left(t_{1}, n\right)=0$, it is enough to see that $\frac{\partial h}{\partial t}(t, n)=-\frac{1}{n} \sin \left(\frac{t}{n}\right) \cos \left(\frac{t_{1}}{2}\right)+\frac{1}{2} \sin \left(\frac{t}{2}\right) \cos \left(\frac{t_{1}}{n}\right) \geq 0$ for $t \in\left[t_{1}, 0\right]$.

Assume first $t_{1} \in\left[-\frac{n \pi}{2}, 0\right]$. As $n \in\left[\frac{2}{3}, 1\right]$ we have $0 \leq-\sin \left(\frac{t}{2}\right) \leq-\sin \left(\frac{t}{n}\right)$ and $0 \leq \cos \left(\frac{t_{1}}{n}\right) \leq \cos \left(\frac{t_{1}}{2}\right)$. Hence $\frac{\partial h}{\partial t}(t, n) \geq 0$.

If $t_{1} \in\left[-n \pi,-\frac{n \pi}{2}\right]$, the study of the signs in the expression of $\frac{\partial h}{\partial t}$ gives directly that this partial is nonnegative.

Finally, we consider the case $t_{1} \in[-\pi,-n \pi]$. As in the former case, studying the signs in the expression of $\frac{\partial h}{\partial t}$ we obtain that $\frac{\partial h}{\partial t}(t, n) \geq 0$ for $t \in[-n \pi, 0]$. Otherwise, it is not difficult to see that $\frac{\partial^{2} h}{\partial n \partial t}(t, n) \geq 0$ for $t \in$ $\left[t_{1},-n \pi\right]$. As $\frac{\partial h}{\partial t}\left(t, \frac{2}{3}\right)=2 \cos \left(\frac{t_{1}}{2}\right) \sin \left(\frac{t}{2}\right)\left(-2 \cos \left(\frac{t}{2}\right)^{2}+\left(\cos \left(\frac{t_{1}}{2}\right)^{2}-\cos \left(\frac{t}{2}\right)^{2}\right)\right)$ $\geq 0$ for $t \in\left[t_{1},-n \pi\right]$, we conclude that $\frac{\partial h}{\partial t}(t, n) \geq 0$.

Our next objective is to prove that $f_{1}(0,-n \pi)<0$. Indeed, making the change of variable $s=t+n \pi / 2$ one has:

$$
\begin{gathered}
f_{1}(0,-n \pi)=\int_{\frac{-n \pi}{2}}^{\frac{n \pi}{2}} \frac{\sin \left(\frac{s}{n}\right)}{\sqrt{-\sin \left(\frac{-n \pi+2 s}{4}\right)\left(\sin \left(\frac{n \pi}{2}\right)+\sin \left(\frac{-n \pi+2 s}{4}\right)\right)}} d s= \\
\int_{0}^{\frac{n \pi}{2}} \sin \left(\frac{s}{n}\right) \frac{\sqrt{\left(\sin \left(\frac{n \pi}{2}\right)-\sin \left(\frac{n \pi+2 s}{4}\right)\right) \sin \left(\frac{n \pi+2 s}{4}\right)}-\sqrt{\left(\sin \left(\frac{n \pi}{2}\right)-\sin \left(\frac{n \pi-2 s}{4}\right)\right) \sin \left(\frac{n \pi-2 s}{4}\right)}}{\sqrt{\left(\sin \left(\frac{n \pi}{2}\right)-\sin \left(\frac{n \pi-2 s}{4}\right)\right) \sin \left(\frac{n \pi-2 s}{4}\right)} \sqrt{\left(\sin \left(\frac{n \pi}{2}\right)-\sin \left(\frac{n \pi+2 s}{4}\right)\right) \sin \left(\frac{n \pi+2 s}{4}\right)}} d s .
\end{gathered}
$$

An easy computation gives us that the numerator in the last integral is always nonpositive, and then $f_{1}(0,-n \pi)<0$. From the definition of $f_{1}$ we also have $f_{1}\left(0,-\frac{n \pi}{2}\right)>0$. Then, taking into account that $\frac{\partial f_{1}}{\partial t_{1}}\left(0, t_{1}\right)>0$, we have that there exists a unique $\left.\widetilde{t}_{1} \in\right]-n \pi,-\frac{n \pi}{2}\left[\operatorname{such}\right.$ that $f_{1}\left(0, \widetilde{t}_{1}\right)=0$. 
We now consider the function $\widetilde{f}_{1}\left(t_{0}\right)=f_{1}\left(t_{0},-t_{0}\right)$, that is the function $f_{1}$ restricted to the side $L_{2}$. Taking into account (6) one has

$$
\frac{\partial \widetilde{f}_{1}}{\partial t_{0}}\left(t_{0}\right)=\frac{\partial f_{1}}{\partial t_{0}}\left(t_{0},-t_{0}\right)-\frac{\partial f_{1}}{\partial t_{1}}\left(t_{0},-t_{0}\right)=-2 \frac{\partial f_{1}}{\partial t_{1}}\left(t_{0},-t_{0}\right)<0 .
$$

According to the definition of $f_{1}$ we have $\tilde{f}_{1}\left(\frac{n \pi}{2}\right)=f_{1}\left(\frac{n \pi}{2},-\frac{n \pi}{2}\right)>0$. Our next purpose is to see that $\widetilde{f}_{1}(n \pi)=f_{1}(n \pi,-n \pi)<0$. Note that

$$
\widetilde{f}_{1}(n \pi)=2 \sqrt{2} \int_{-n \pi}^{0} \frac{\cos \left(\frac{t}{n}\right)}{\sqrt{\cos (t)-\cos (n \pi)}} .
$$

A direct computation gives

$$
\begin{aligned}
& \tilde{f}_{1}(n \pi) \\
& =2 \sqrt{2} \int_{0}^{\frac{n \pi}{2}} \frac{\sin \left(\frac{s}{n}\right)\left(\sqrt{\cos \left(s+\frac{n \pi}{2}\right)-\cos (n \pi)}-\sqrt{\cos \left(s-\frac{n \pi}{2}\right)-\cos (n \pi)}\right)}{\sqrt{\cos \left(s+\frac{n \pi}{2}\right)-\cos (n \pi)} \sqrt{\cos \left(s-\frac{n \pi}{2}\right)-\cos (n \pi)}} .
\end{aligned}
$$

It is not hard to see that the numerator in the above integrand is nonpositive, in particular $\widetilde{f}_{1}(n \pi)<0$.

Therefore there exists $\left.\widetilde{t}_{0} \in\right] \frac{n \pi}{2}, n \pi\left[\right.$ such that $f_{1}\left(\widetilde{t}_{0},-\widetilde{t}_{0}\right)=0, f_{1}$ is positive in $\left\{\left(t_{0},-t_{0}\right) \in T \mid 0<t_{0}<\widetilde{t}_{0}\right\}$ and negative in $\left\{\left(t_{0},-t_{0}\right) \in T \mid \widetilde{t}_{0}<t_{0}<\pi\right\}$.

Now, we prove that $\lim _{t_{1} \rightarrow-\pi} f_{1}\left(t_{0}, t_{1}\right)=-\infty$. In order to do this we consider a new set of parameters

$$
s\left(t_{0}, t_{1}\right)=\sin \left(\frac{t_{0}}{2}\right), \quad r\left(t_{0}, t_{1}\right)=\sin \left(\frac{t_{1}}{2}\right) .
$$

Note that $r\left(t_{0},-\pi\right)=-1$. Our next objective is to see that $\lim _{r \rightarrow-1} f_{1}(s, r)$ $=-\infty$. In order to do this, we derive again the 1-form $\Phi$ defined in Step 1 and we obtain the following equality:

$$
\frac{\partial^{4} \Phi}{\partial r^{4}}+b_{3} \frac{\partial^{3} \Phi}{\partial r^{3}}+b_{2} \frac{\partial^{2} \Phi}{\partial r^{2}}+b_{1} \frac{\partial \Phi}{\partial r}+b_{0} \Phi=d(\varphi),
$$

where

$$
\begin{aligned}
& b_{3}=\frac{2\left(5 r^{3}+2 s-8 r^{2} s+r\left(-2+3 s^{2}\right)\right)}{\left(-1+r^{2}\right)(r-s)^{2}} \\
& b_{2}=\frac{-16(r-s)^{2}+n^{2}\left(-8+99 r^{2}-116 r s+25 s^{2}\right)}{4 n^{2}\left(-1+r^{2}\right)(r-s)^{2}} \\
& b_{1}=\frac{\left(-48+57 n^{2}\right) r+\left(48-39 n^{2}\right) s}{4 n^{2}\left(-1+r^{2}\right)(r-s)^{2}} \\
& b_{0}=\frac{3\left(n^{2}-4\right)}{4 n^{2}\left(-1+r^{2}\right)(r-s)^{2}}
\end{aligned}
$$


and $\varphi$ is the following meromorphic function:

$$
\varphi(u)=\frac{u^{\frac{2}{n}+1}\left(a^{\prime} u^{4}+b^{\prime} u^{3}+c^{\prime} u^{2}+d^{\prime} u+e^{\prime}\right)\left(u^{2}-1-2 s \mathrm{i} u\right)^{\frac{1}{2}}}{4 n^{2}\left(-1+r^{2}\right)(r-s)^{2}\left(u^{2}-1-2 r \mathrm{i} u\right)^{\frac{7}{2}}},
$$

with $a^{\prime}=-3 n(2+n), b^{\prime}=-3 \operatorname{in}((-4+3 n) r-(4+5 n) s), c^{\prime}=12 n(1+2 r s)$, $d^{\prime}=-3 \mathrm{i} n((4+3 n) r+(4-5 n) s)$ and $e^{\prime}=3 n(n-2)$. Integrating by parts in (11), we have that $f_{1}$ is a solution of the fourth order ordinary differential equation given by

$$
\frac{\partial^{4} f_{1}}{\partial r^{4}}+b_{3} \frac{\partial^{3} f_{1}}{\partial r^{3}}+b_{2} \frac{\partial^{2} f_{1}}{\partial r^{2}}+b_{1} \frac{\partial f_{1}}{\partial r}+b_{0} f_{1}=0
$$

Observe that this equation presents a regular singular point in $r=-1$ and then we can use the Frobenius method to compute the limit of $f_{1}$ when $r$ tends to -1 (see $\S 4.8$ in $[\mathbf{3}]$ ). Taking into account the coefficients of Equation (12) and the aforementioned method, we deduce that

$f_{1}(s, r)=c_{1} \log (1+r) \phi_{1}(s, r)+c_{2} \phi_{2}(s, r)+c_{3}(r+1) \phi_{3}(s, r)+c_{4}(r+1)^{2} \phi_{4}(s, r)$,

where $c_{i} \in \mathbb{R}, \phi_{1}(s,-1) \neq 0$ and $\phi_{i}$ are analytic at the points $(s,-1), i \in$ $\{1,2,3,4\}$. A direct computation using (8) and (10) proves that $\lim _{r \rightarrow-1} \frac{\partial f_{1}}{\partial r}$ $=+\infty$. Thus $c_{1} \neq 0$ and $\lim _{r \rightarrow-1} f_{1}(s, r)=-\infty$.

Step 3. With regard to $C_{2}$, we shall check that $C_{2} \cap\left\{\left(0, t_{1}\right) \in T \mid \widetilde{t}_{1} \leq t_{1}<\right.$ $0\}=\emptyset$ and that if $\left(\widehat{t_{0}},-\widehat{t}_{0}\right)$ is a critical point of $C_{2}$ in $L_{2}$, then $\widehat{t}_{0}>\widetilde{t}_{0}$.

Clearly, from the definition of $f_{2}$ one has $f_{2}\left(0, t_{1}\right)<0$ for $t_{1} \in[-n \pi, 0[$. Thus we obtain the first assertion in the present step.

In order to prove the second one we need an apropriate expression for $\frac{\partial f_{2}}{\partial t_{0}}$. Observe that

$$
\frac{\partial \Phi}{\partial t_{0}}=u^{2 / n} \tau-\frac{2 a}{n} u^{2 / n-1} \psi d u+a d\left(u^{2 / n} \psi\right)
$$

where

$$
\begin{aligned}
\tau & =\frac{-\mathrm{i}\left(-1+\mathrm{e}^{\mathrm{i} \frac{t_{0}}{2}} u\right)}{4\left(1+\mathrm{e}^{\mathrm{i} \frac{t_{0}}{2}} u\right)} \frac{d u}{v}, \\
\psi & =\frac{\left(u+\mathrm{e}^{-\mathrm{i} \frac{t_{1}}{2}}\right)\left(u-\mathrm{e}^{\mathrm{i} \frac{t_{1}}{2}}\right)}{v}, \\
a & =\frac{1}{4\left(\sin \left(\frac{t_{1}}{2}\right)-\sin \left(\frac{t_{0}}{2}\right)\right)} .
\end{aligned}
$$


Integrating by parts once again in the above equality and computing the real part, we get:

$$
\begin{aligned}
\frac{\partial f_{2}}{\partial t_{0}}\left(t_{0}, t_{1}\right)= & \int_{t_{1}}^{t_{0}}\left(\frac{\cos \left(\frac{t}{n}\right) \sin \left(\frac{t}{2}\right)}{n v\left(t, t_{0}, t_{1}\right)\left(\sin \left(\frac{t_{0}}{2}\right)-\sin \left(\frac{t_{1}}{2}\right)\right)}+\frac{\sin \left(\frac{t}{n}\right) \tan \left(\frac{t+t_{0}}{4}\right)}{4 v\left(t, t_{0}, t_{1}\right)}\right) d t \\
& -\frac{\sin \left(\frac{t_{1}}{2}\right)}{n\left(\sin \left(\frac{t_{0}}{2}\right)-\sin \left(\frac{t_{1}}{2}\right)\right)} f_{1}\left(t_{0}, t_{1}\right) .
\end{aligned}
$$

In particular, if $t_{1}=-t_{0}$ one has that the integral of the first summand vanishes, and so

$$
\frac{\partial f_{2}}{\partial t_{0}}\left(t_{0},-t_{0}\right)=\int_{-t_{0}}^{t_{0}} \frac{\sin \left(\frac{t}{n}\right) \tan \left(\frac{t+t_{0}}{4}\right)}{4 v\left(t, t_{0}, t_{1}\right)} d t+\frac{1}{2 n} f_{1}\left(t_{0},-t_{0}\right) .
$$

Taking into account that $\widetilde{t}_{0}<n \pi$, it is not difficult to see that

$$
\int_{-t_{0}}^{t_{0}} \frac{\sin \left(\frac{t}{n}\right) \tan \left(\frac{t+t_{0}}{4}\right)}{4 v\left(t, t_{0}, t_{1}\right)} d t>0
$$

for $0<t_{0} \leq \widetilde{t}_{0}$, and so $\frac{\partial f_{2}}{\partial t_{0}}\left(t_{0},-t_{0}\right)$ is positive in the points where the function $f_{1}$ is nonnegative. This concludes the assertion.

By Steps 1, 2 and 3 we deduce that there are no points $\left(t_{0}, t_{1}\right)$ in $T$ with $f_{1}\left(t_{0}, t_{1}\right) \geq 0$ and $f_{2}\left(t_{0}, t_{1}\right)=0$ apart from the points $\left\{\left(t_{0},-t_{0}\right) \mid 0 \leq t_{0} \leq\right.$ $\left.\widetilde{t}_{0}\right\}$.

Corollary 7. $M$ is invariant under the antiholomorphic involution $S_{v}(u)=$ $\bar{u}$ which corresponds to the reflection in the plane $\left\{x_{1}=0\right\}$.

\section{The existence results.}

In the former section we have seen that if $X: M \longrightarrow \mathbb{R}^{3}$ is a solution of Problem 1 satisfying $X(M) \subset\left(\left(S \backslash \mathcal{E}\left(\Gamma_{\theta d}\right)\right) \cup \Gamma_{\theta d}\right)$ then $M$ is biholomorphic to $\mathcal{N}^{\prime}=\overline{\mathcal{U}_{t_{0}}^{-t_{0}}}$ and their Weierstrass data are given by

$$
g=\mathrm{i} u^{\frac{2}{n}}, \quad \Phi_{3}=\lambda \frac{d u}{v}, \quad v=\sqrt{u^{4}-2 u^{2} \cos \left(t_{0}\right)+1}, \quad n \in\left[\frac{2}{3}, 1\right] .
$$

At this point, we observe that, up to an easy conformal transformation, the above Weierstrass data for $n \in[1,2[$ correspond to a López-Martín example, it is to say, solutions of Problem 1 verifying $X(M) \subset \mathcal{E}\left(\Gamma_{\theta d}\right)$. For the existence of this examples we refer the reader to [10].

Moreover, if $n=2$ then the surface is a Jenkins-Serrin graph. Extension by Schwarz reflection of these surfaces gives embedded doubly periodic examples with two orthogonal planes of symmetry between adjacent saddle towers. These examples were studied by H. Karcher in [7].

Therefore, to complete the existence part of the Main Theorem it suffices to prove that indeed for $n \in\left[\frac{2}{3}, 1\right]$ the above conformal representation leads 


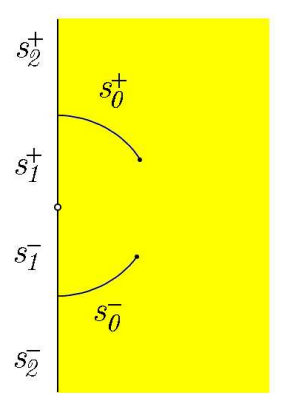

a)

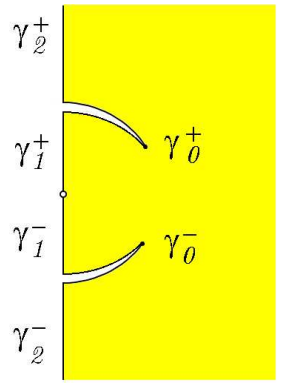

b)

Figure 5. a) The domain $u(M)$. b) The surface $M$.

to a solution of Problem 1 which lies in the exterior of the convex hull of its boundary and this is the purpose of the present section.

In what follows we denote $M=\mathcal{N}^{\prime}$ and $\gamma_{i}^{+}=u^{-1}\left(s_{i}^{+}\right), \gamma_{i}^{-}=u^{-1}\left(s_{i}^{-}\right)$, $i=0,1,2$, where $s_{i}^{+}$and $s_{i}^{-}$are those defined in Section 2 corresponding to $t_{1}=-t_{0}$ for $i=0,1,2$ (see Figure 5). Moreover, we label $\gamma^{+}=\cup_{i=0}^{2} \gamma_{i}^{+}$ and $\gamma^{-}=\cup_{i=0}^{2} \gamma_{i}^{-}$. We use the notation $\ell_{i}^{ \pm}$introduced in Section 1 for the half-lines in the polygonal $\Gamma_{\theta d}$. Furthermore, recall that the set of Cartesian coordinates was introduced at the beginning of Section 2.

Now, we consider the curve $\gamma_{0}^{+}$which consists of two copies, $\delta_{1}^{+}$and $\delta_{2}^{+}$, of $s_{0}^{+}$. We can assume that $\delta_{1}^{+}(t)$ and $\delta_{2}^{+}(t)$ are the two lifts to $M$ of the curve $\mathrm{e}^{\mathrm{i} \frac{t}{2}}, t \in\left[t_{0}, \pi\right]$, in the $u$-plane, satisfying $\delta_{1}^{+}(\pi) \in \gamma_{1}^{+}$and $\delta_{2}^{+}(\pi) \in \gamma_{2}^{+}$, respectively. Define $\widetilde{h}:] 0, \pi\left[\longrightarrow \mathbb{R}\right.$ as $\widetilde{h}\left(t_{0}\right)=2 \operatorname{Re}\left(\int_{\delta_{2}^{+}} \tau\right)$, where $\tau=\frac{d u}{v}$. A direct computation gives $\widetilde{h}\left(t_{0}\right)=\frac{\sqrt{2}}{2} \int_{t_{0}}^{\pi} \frac{d t}{\sqrt{\cos \left(t_{0}\right)-\cos (t)}}>0$. As we are assuming that the immersion $X: M \longrightarrow \mathbb{R}^{3}$ is normalized so that the distance between the planes $\pi_{1}$ and $\pi_{2}$ is 1 , we have $\lambda=\frac{1}{\widetilde{h}\left(t_{0}\right)}$.

As usual, we define $\left(\Phi_{1}, \Phi_{2}, \Phi_{3}\right)=\frac{1}{2}\left(-\mathrm{i}\left(u^{-\frac{2}{n}}+u^{\frac{2}{n}}\right), u^{-\frac{2}{n}}-u^{\frac{2}{n}}, 1\right) \Phi_{3}$. Since $M$ is homeomorphic to a closed disk minus two boundary points, we have that $X: M \longrightarrow \mathbb{R}^{3}$ given by $X(p)=\int_{p_{0}}^{p}\left(\Phi_{1}, \Phi_{2}, \Phi_{3}\right)$ is a well-defined conformal minimal immersion verifying Condition (1) in the statement of Problem 1. Let us see that $\left(M, g, \Phi_{3}\right)$ fulfill also the other conditions.

Denote $S_{h}, S_{v}$ the antiholomorphic transformations on $M$ given by $S_{h}((u, v))=\left(1 / \bar{u}, \bar{v} / \bar{u}^{2}\right), S_{v}((u, v))=(\bar{u}, \bar{v})$. Observe that the point $P_{0}=$ $\left(1,2 \sin \left(\frac{t_{0}}{2}\right)\right)$ is invariant under $S_{h}$ and $S_{v}$. Moreover, we have

$$
g \circ S_{h}=1 / \bar{g}, \quad g \circ S_{v}=-\bar{g}, \quad S_{h}^{*}\left(\phi_{3}\right)=-\overline{\phi_{3}}, \quad S_{v}^{*}\left(\phi_{3}\right)=\overline{\phi_{3}} .
$$

Hence elementary arguments imply that $S_{h}$ (resp. $S_{v}$ ) induces on $X(M)$ a symmetry with respect to the plane $\left\{x_{3}=0\right\}$ (resp. $\left.\left\{x_{1}=0\right\}\right)$. 
First, notice that

$$
S_{v}\left(\gamma_{i}^{+}\right)=\gamma_{i}^{-}, i=0,1,2
$$

$$
S_{h}\left(\gamma_{1}^{+}\right)=\gamma_{2}^{+}, S_{h}\left(\gamma_{1}^{-}\right)=\gamma_{2}^{-}, S_{h}\left(\gamma_{0}^{+}\right)=\gamma_{0}^{+}, S_{h}\left(\gamma_{0}^{-}\right)=\gamma_{0}^{-} .
$$

It is straightforward to check that the conditions on the boundary are satisfied. Actually, we have:

Lemma 6. The maps $\left.X\right|_{\gamma^{+}},\left.X\right|_{\gamma^{-}}$are injective, $X\left(\gamma_{i}^{+}\right)=\ell_{i}^{+}$and $X\left(\gamma_{i}^{-}\right)=$ $\ell_{i}^{-}$, for $i=0,1,2$, it is to say, $X(\partial(M))=\Gamma_{\theta d}$ for $\theta=(3 n-2) \pi / n$.

Taking into account Lemma 6, the expression of the oriented distance $d:] 0, \pi\left[\longrightarrow \mathbb{R}\right.$ between $\ell_{0}^{+}$and $\ell_{0}^{-}$is $d\left(t_{0}\right)=\operatorname{Re}\left(\int_{\widehat{\delta}} \Phi_{1}\right)$, where now $\widehat{\delta}$ is the lift of the oriented curve $\mathrm{e}^{\mathrm{i} \frac{t}{2}}, t \in\left[-t_{0}, t_{0}\right]$, in the $u$-plane. Since $\left(S_{h}\right)_{*}(\widehat{\delta})=\widehat{\delta}$, $\left(S_{v}\right)_{*}(\widehat{\delta})=-\widehat{\delta}$ and taking into account (13) we deduce that $\int_{\widehat{\delta}} \Phi_{1}=\overline{\int_{\widehat{\delta}} \Phi_{1}}=$ $\frac{\lambda}{4} \widetilde{f}_{1}\left(t_{0}\right)$, where, as in Section $2, \Phi=\frac{u^{\frac{2}{n}}}{v} d u$ and $\widetilde{f}_{1}\left(t_{0}\right)=f_{1}\left(t_{0},-t_{0}\right)$. Hence we have

$$
d\left(t_{0}\right)=\frac{1}{4} \frac{\widetilde{f}_{1}\left(t_{0}\right)}{\widetilde{h}\left(t_{0}\right)} .
$$

A thoughtful study of the function $d$ will be very useful in order to prove the rest of the conditions on the immersion $X: M \longrightarrow \mathbb{R}^{3}$. In this context we shall see:

Lemma 7. The function $d:] 0, \pi[\rightarrow \mathbb{R}$ satisfies:

1. It vanishes at only one point $\left.\widetilde{t}_{0} \in\right] \frac{n \pi}{2}, n \pi[$. Furthermore, $d$ is positive in $] 0, \widetilde{t}_{0}[$ and negative in $] \widetilde{t}_{0}, \pi[$.

2. $\lim _{t_{0} \rightarrow 0} d\left(t_{0}\right)=0$. In particular, $d$ is bounded in $] 0, \widetilde{t}_{0}[$.

3. It has only a critical point $\left.t_{0}^{\prime} \in\right] 0, \widetilde{t}_{0}[$ which is a maximum. In particular, $\left.\sharp\left[d^{-1}(\{x\})\right]=2, \forall x \in\right] 0, d\left(\widetilde{t}_{0}\right)[$.

Proof. We had seen (see Step 2 in Proposition 6) that there exists a unique $\left.\widetilde{t}_{0} \in\right] \frac{n \pi}{2}, n \pi\left[\right.$ such that $\widetilde{f}_{1}\left(\widetilde{t}_{0}\right)=0, \widetilde{f}_{1}\left(t_{0}\right)>0$ in $] 0, \widetilde{t}_{0}\left[\right.$ and $\widetilde{f}_{1}\left(t_{0}\right)<0$ in ]$\widetilde{t}_{0}, \pi[$. Note that this proves the first assertion.

In order to prove the second statement, observe that

$$
\lim _{t_{0} \rightarrow 0} \tilde{f}_{1}\left(t_{0}\right)=\lim _{t_{0} \rightarrow 0} \sqrt{2} \int_{-1}^{1} \frac{t_{0} \cos \left(\frac{t_{0} s}{n}\right)}{\sqrt{\cos \left(t_{0} s\right)-\cos \left(t_{0}\right)}} d s=2 \int_{-1}^{1} \frac{d s}{\sqrt{1-s^{2}}}=2 \pi .
$$

Moreover, it is clear that $0 \leq \sqrt{\cos \left(t_{0}\right)-\cos (t)} \leq \sqrt{1-\cos (t)}, t \in\left[t_{0}, \pi\right]$, then $\widetilde{h}\left(t_{0}\right) \geq \frac{\sqrt{2}}{2} \int_{t_{0}}^{\pi} \frac{d t}{\sqrt{1-\cos (t)}}=-\log \left[\tan \left(\frac{t_{0}}{4}\right)\right]$, and so

$$
\lim _{t_{0} \rightarrow 0} \widetilde{h}\left(t_{0}\right) \geq \lim _{t_{0} \rightarrow 0}\left(-\log \left[\tan \left(\frac{t_{0}}{4}\right)\right]\right)=+\infty .
$$


Both (17) and (18) give Assertion 2. Concerning Assertion 3, we shall prove that the functions $\tilde{f}_{1}, \widetilde{h}$ satisfy the following differential equations:

$$
\begin{aligned}
\widetilde{f}_{1}^{\prime \prime}\left(t_{0}\right)+\cot \left(t_{0}\right) \widetilde{f}_{1}^{\prime}\left(t_{0}\right)+\frac{4-n^{2}}{4 n^{2}} \widetilde{f}_{1}\left(t_{0}\right) & =0, \\
\widetilde{h}^{\prime \prime}\left(t_{0}\right)+\cot \left(t_{0}\right) \widetilde{h}^{\prime}\left(t_{0}\right)-\frac{1}{4} \widetilde{h}\left(t_{0}\right) & =0 .
\end{aligned}
$$

The above ordinary differential equations can be obtained from the following equalities integrating by parts:

$$
\frac{\partial^{2} \Phi}{\partial t_{0}^{2}}+\cot \left(t_{0}\right) \frac{\partial \Phi}{\partial t_{0}}+\frac{4-n^{2}}{4 n^{2}} \Phi=d(\widetilde{G}), \quad \frac{\partial^{2} \tau}{\partial t_{0}^{2}}+\cot \left(t_{0}\right) \frac{\partial \tau}{\partial t_{0}}-\frac{1}{4} \tau=d(\widetilde{H}),
$$

where

$$
\widetilde{G}(u)=\frac{u^{\frac{2+n}{n}}\left(n\left(-1+u^{4}\right)+2\left(1+u^{4}\right)-4 u^{2} \cos \left(t_{0}\right)\right)}{4 n\left(1+u^{4}-2 u^{2} \cos \left(t_{0}\right)\right)^{\frac{3}{2}}}
$$

and

$$
\widetilde{H}(u)=\frac{u\left(-1+u^{4}\right)}{4\left(1+u^{4}-2 u^{2} \cos \left(t_{0}\right)\right)^{\frac{3}{2}}} .
$$

Let $t_{0}^{\prime}$ a critical point of $d$ in $] 0, \widetilde{t}_{0}\left[\right.$. This implies that $d^{\prime}\left(t_{0}^{\prime}\right)=0$ and so $\left(\widetilde{f}_{1}^{\prime} \widetilde{h}-\widetilde{f}_{1} \widetilde{h}^{\prime}\right)\left(t_{0}^{\prime}\right)=0$. Therefore we have the following expression for the second derivative of $d$ at the point $t_{0}^{\prime}$ :

$$
d^{\prime \prime}\left(t_{0}^{\prime}\right)=\frac{1}{4} \frac{\widetilde{f}_{1}^{\prime \prime} \widetilde{h}-\widetilde{f}_{1} \widetilde{h}^{\prime \prime}}{\widetilde{h}^{2}}\left(t_{0}^{\prime}\right) .
$$

Hence, using (19) and (20), we obtain that $d^{\prime \prime}\left(t_{0}^{\prime}\right)=-\cot \left(t_{0}\right) d^{\prime}\left(t_{0}^{\prime}\right)-\frac{1}{n^{2}} d\left(t_{0}^{\prime}\right)$ $<0$. Consequently, there exists only one critical point of $d, t_{0}^{\prime}$, in $] 0, \widetilde{t}_{0}[$ and it is a maximum. Obviously, $d\left(t_{0}^{\prime}\right)=\operatorname{Maximum}\left\{d\left(t_{0}\right) \mid t_{0} \in\right] 0, \widetilde{t}_{0}[\}$.

Hence, it is clear that $\left.\sharp\left[d^{-1}(\{x\})\right] \geq 2, \forall x \in\right] 0, d\left(t_{0}^{\prime}\right)\left[\right.$. If $\sharp\left[d^{-1}(\{x\})\right]>2$, for some $x \in] 0, d\left(t_{0}^{\prime}\right)[$, then it implies the existence of a local minimum of $d$ in $] 0, \widetilde{t}_{0}[$, which is absurd. This concludes the proof.

Remark 2. For each $n \in\left[\frac{2}{3}, 1\right]$, we denote either by $d_{n}$ or by $d_{\theta}^{\prime}$ the maximum of the distance function $\left.\left.d\left(t_{0}\right), t_{0} \in\right] 0, \widetilde{t}_{0}\right]$. Observe that the function $d\left(n, t_{0}\right)$ is a differentiable function on $\left.\left[\frac{2}{3}, 1\right] \times\right] 0, \pi\left[\right.$. Let us check that $\frac{\partial d}{\partial n}>0$.

Taking into account that $\widetilde{t_{0}}<n \pi$ and the definition of the function $\widetilde{f}_{1}$, we obtain

$$
\frac{\partial d}{\partial n}=\frac{1}{4 \widetilde{h}} \frac{\partial \widetilde{f}_{1}}{\partial n}=\frac{\sqrt{2}}{4 n^{2} \widetilde{h}} \int_{-t_{0}}^{t_{0}} \frac{t \sin \left(\frac{t}{n}\right)}{\sqrt{\cos (t)-\cos \left(t_{0}\right)}}>0 .
$$

Then $n \rightarrow d_{n}$ is a continuous increasing function in $\left[\frac{2}{3}, 1\right]$, equivalently the function $\theta \rightarrow d_{\theta}^{\prime}$ is increasing in $[0, \pi]$. Therefore, $d_{\theta_{0}}^{\prime}<d_{\theta_{1}}^{\prime}$ for $0 \leq \theta_{0}<$ $\theta_{1} \leq \pi$. 
At this point, we recall that the distance function for the examples which lie in the convex hull of their boundary coincides with our function $d$ for $n \in[1,2]$ (see [10]). As $\frac{\partial d}{\partial n}>0$ we have that $d_{n_{0}}<d_{n_{1}}$ for $\frac{2}{3} \leq n_{0}<n_{1} \leq 2$. Hence, we infer that $d_{\theta}^{\prime}<d_{\theta}$, for $\theta \in[0, \pi]$, where $d_{\theta}$ is the maximum of the distance function for the López-Martín examples (see Remark 4 in [10]).

From the asymptotic expansion of the Weierstrass data we have that $X$ can be expressed locally around $E_{1}$ as

$$
\begin{aligned}
X(u) & =\left(X_{1}(u), X_{2}(u), X_{3}(u)\right) \\
& =\left(-\mathrm{i} \frac{n \lambda}{2(2-n)} \bar{u}^{1-\frac{2}{n}}\left(1+O_{1}(u)+\mathrm{i} O_{2}(u)\right), O_{3}(u)\right),
\end{aligned}
$$

where $O_{i}(u) /|u|$ is a bounded function in a neighbourhood of $E_{1}, i=1,2,3$. Using this fact and Lemma 2.1 in [12] it is not difficult to prove the following lemma:

Lemma 8. The minimal immersion $X: M \rightarrow \mathbb{R}^{3}$ is proper and $X(M)$ is contained in the slab $S$.

Let us consider $M_{1}=\{(u, v) \in M \quad|u| \leq 1\}$ and $M_{2}=\{(u, v) \in$ $M \quad|u| \geq 1\}$. We recall that we had denoted by $\widehat{\delta}$ and $\delta_{i}^{+}$the lifts to $M$ of the curves of $u(M)$ given by $\mathrm{e}^{\mathrm{i} \frac{t}{2}}$, for $t \in\left[-t_{0}, t_{0}\right]$ and $t \in\left[t_{0}, \pi\right]$, respectively satisfying $\delta_{i}^{+}(\pi) \in \gamma_{i}^{+}, i=1,2$. Clearly, the surfaces $M_{1}$ and $M_{2}$ are topologically a closed disk minus one boundary point. Moreover, $M=M_{1} \cup M_{2}, \quad M_{1} \cap M_{2}=\widehat{\delta}$ and $\partial\left(M_{1}\right)=\widehat{\delta} \cup \gamma_{1}^{+} \cup \gamma_{1}^{-} \cup \delta_{1}^{+} \cup \delta_{1}^{-}, \quad \partial\left(M_{2}\right)=$ $\widehat{\delta} \cup \gamma_{2}^{+} \cup \gamma_{2}^{-} \cup \delta_{2}^{+} \cup \delta_{2}^{-}$, where $\delta_{i}^{-}=S_{v}\left(\delta_{i}^{+}\right)$for $i=1,2$.

Our next objective is to prove the following assertion:

Claim 4. $\left.X\right|_{\widehat{\delta}}$ is injective and $X(\widehat{\delta}) \subset\left\{x_{2} \leq 0, x_{3}=0\right\}$.

Proof. To see this we observe that $X_{2}(\hat{\delta}(t))=X_{2}(\hat{\delta}(t))-X_{2}\left(\hat{\delta}\left(-t_{0}\right)\right)=$ $-\sqrt{2} \int_{-t_{0}}^{t} \frac{\sin (s / n)}{\sqrt{\cos (s)-\cos \left(t_{0}\right)}} d s$. Since $0<t_{0} \leq n \pi$ we have that $X_{2} \circ \widehat{\delta}$ is a nonpositive decreasing function for $t \in\left[-t_{0}, 0\right]$. This fact and the vertical symmetry $S_{v}$ prove the assertion.

Let us denote $M^{+}=\{(u, v) \in M \mid \operatorname{Im}(u) \geq 0\}$ and $M^{-}=\{(u, v) \in M$ $\operatorname{Im}(u) \leq 0\}$, and define $\rho$ as the lift to $M$ of the divergent curve $] 0,+\infty[$ in the $u$-plane. We parametrize $\rho$ as follows: $\left.\rho(t)=u^{-1}(t), t \in\right] 0,+\infty[$.

Obviously, the surfaces $M^{+}$and $M^{-}$are topologically a closed disk minus two boundary points. Furthermore, $M=M^{+} \cup M^{-}, M^{+} \cap M^{-}=\rho$ and $\partial\left(M^{+}\right)=\rho \cup \gamma_{1}^{+} \cup \gamma_{2}^{+} \cup \gamma_{0}^{+}, \partial\left(M^{-}\right)=\rho \cup \gamma_{1}^{-} \cup \gamma_{2}^{-} \cup \gamma_{0}^{-}$. Next we prove:

Claim 5. $X(\rho(t)) \subset\left\{\left(x_{1}, x_{2}, x_{3}\right) \mid x_{1}=0, x_{2} \leq-\varepsilon,-\frac{1}{2}<x_{3}<\frac{1}{2}\right\}$, where $\varepsilon=-X_{2}\left(P_{0}\right)>0$. 
Proof. Taking the symmetry $S_{h}$ into account, it suffices to prove the assertion for $X(\rho(] 0,1]))$. From the Weierstrass data we deduce that

$$
X_{2}(\rho(t))=-\varepsilon-\int_{t}^{1} \frac{s^{-\frac{2}{n}}-s^{\frac{2}{n}}}{\sqrt{s^{4}-2 \cos \left(t_{0}\right) s^{2}+1}} d s .
$$

Using Claim 4 we have $\varepsilon=-X_{2}\left(P_{0}\right)>0$. So $X_{2} \circ \rho$ is decreasing and nonpositive in ]0,1].

Moreover, taking (21) and the symmetry $S_{h}$ into account, we obtain that

$$
\lim _{t \rightarrow 0} X_{2}(\rho(t))=\lim _{t \rightarrow+\infty} X_{2}(\rho(t))=-\infty .
$$

At this point we can prove the following lemma:

Lemma 9. The minimal immersion $X: M \longrightarrow \mathbb{R}^{3}$ verifies:

1. $X(M) \subset\left(\left(S \backslash \mathcal{E}\left(\Gamma_{\theta d}\right)\right) \cup \Gamma_{\theta d}\right)$.

2. The surfaces $X\left(M_{1} \backslash\left(\delta_{1}^{+} \cup \delta_{1}^{-}\right)\right)$and $X\left(M_{2} \backslash\left(\delta_{2}^{+} \cup \delta_{2}^{-}\right)\right)$are graphs on the plane $\left\{x_{3}=0\right\}$.

3. $d>0$ implies that $X$ is an embedding. If $d=0$, then $\left.X\right|_{M \backslash \gamma^{+}}$and $\left.X\right|_{M \backslash \gamma^{-}}$are injective.

Proof. Denote by $\mathfrak{p}_{3}$ the orthogonal projection on the plane $\left\{x_{3}=0\right\}$. Using (21) once again and the symmetries, it is not hard to see that $X\left(M^{+}\right)$ and $X\left(M^{-}\right)$are contained in a wedge of the slab $S$. Then we can apply Statement (ii) in Theorem 1 to conclude that $X\left(M^{+}\right)$and $X\left(M^{-}\right)$lies in the convex hull of their boundary.

In case $d=0$, taking into account Lemma 6, Claim 5 and the interior maximum principle we have the proof of Assertion 1. Moreover, in this case the interior maximum principle also gives us that $X\left(M^{+}\right) \cap\left\{\left(x_{1}, x_{2}, x_{3}\right) \in\right.$ $\left.\mathbb{R}^{3} \mid x_{1}=0,-\varepsilon<x_{2}<0\right\}=\emptyset$, and so, taking into account the symmetry $S_{v}$, we deduce that

$$
\mathfrak{p}_{3}(X(M)) \cap\left\{\left(x_{1}, x_{2}, x_{3}\right) \in \mathbb{R}^{3} \mid x_{1}=0,-\varepsilon<x_{2}<0\right\}=\emptyset .
$$

In case $d>0$ the above reasoning implies

$$
X(M) \subset S \backslash\left\{\left(x_{1}, x_{2}, x_{3}\right) \in \mathbb{R}^{3} \mid x_{1}=0, x_{2}>0\right\} .
$$

Next, we prove Assertion 1 for $d>0$ and Assertion 2. In what follows we denote $\alpha=\mathfrak{p}_{3}\left(\partial\left(X\left(M_{1}\right)\right)\right)$. We also introduce the following notation:

(A) If $d=0, W_{0}$ will denote the bounded connected component of $\mathbb{R}^{2} \backslash$ $\alpha$, whereas $W_{1}$ will denote the unbounded connected component which is disjoint from $\mathfrak{p}_{3}\left(\mathcal{E}\left(\Gamma_{\theta d}\right)\right)$.

(B) If $d>0$, observe that $\alpha$ is a connected simple curve. In this case we denote $W_{0}$ as the connected component of $\mathbb{R}^{2} \backslash \alpha$ containing the point $(0,0)$ and $W_{1}$ the other one. 
In both cases $\mathfrak{p}_{3}\left(M_{1}\right) \subset W_{0} \cup W_{1} \cup \alpha$. Since $\mathfrak{p}_{3}\left(X\left(\partial\left(M_{1}\right)\right)\right)=\alpha$ and $\mathfrak{p}_{3} \circ X$ is proper on $M_{1}$ (see Lemma 8), then $\mathfrak{p}_{3} \circ X\left(M_{1} \backslash \partial\left(M_{1}\right)\right) \cap W_{0}$ is closed in $W_{0}$. Furthermore, $g\left(M_{1} \backslash \partial\left(M_{1}\right)\right) \cap \mathbb{S}^{1}=\emptyset$ and so $\left.\left(\mathfrak{p}_{3} \circ X\right)\right|_{M_{1} \backslash \partial\left(M_{1}\right)}$ is a local diffeomorphism. In particular, $\mathfrak{p}_{3} \circ X\left(M_{1} \backslash \partial\left(M_{1}\right)\right) \cap W_{0}$ is an open set of $W_{0}$. Hence we deduce that either $\mathfrak{p}_{3} \circ X\left(M_{1} \backslash \partial\left(M_{1}\right)\right) \cap W_{0}=\emptyset$ or $\mathfrak{p}_{3} \circ X\left(M_{1} \backslash \partial\left(M_{1}\right)\right) \cap W_{0}=W_{0}$. According to (22) and (23) we have that $\mathfrak{p}_{3} \circ X\left(M_{1} \backslash \partial\left(M_{1}\right)\right) \cap W_{0}=\emptyset$. A similar argument yields $\mathfrak{p}_{3}\left(X\left(M_{1}\right)\right) \cap W_{1}=$ $W_{1}$, i.e., $\mathfrak{p}_{3}\left(X\left(M_{1}\right)\right)=W_{1} \cup \alpha$. Hence, using the symmetry $S_{h}$ and the interior maximum principle we conclude the proof of Assertion 1.

From the above reasoning we have that $\mathfrak{p}_{3} \circ X: M_{1} \backslash\left(\delta_{1}^{+} \cup \delta_{1}^{-}\right) \longrightarrow W_{1} \cup \alpha$ is a proper local diffeomorphism and so $\mathfrak{p}_{3} \circ X$ is a covering map. Since $\left.\left(\mathfrak{p}_{3} \circ X\right)\right|_{\gamma_{1}^{+}}$is one-to-one we obtain that $X\left(M_{1} \backslash\left(\delta_{1}^{+} \cup \delta_{1}^{-}\right)\right)$is a graph on the plane $\left\{x_{3}=0\right\}$.

Using that $\partial\left(X\left(M_{1}\right)\right) \subset\left\{\left(x_{1}, x_{2}, x_{3}\right) \in \mathbb{R}^{3} \mid 0 \leq x_{3} \leq \frac{1}{2}\right\}$ and Lemma 2.1 in $[\mathbf{1 2}]$ we infer that

$$
X\left(M_{1}\right) \subset\left\{\left(x_{1}, x_{2}, x_{3}\right) \in \mathbb{R}^{3} \mid 0 \leq x_{3} \leq \frac{1}{2}\right\} .
$$

Then, taking into account the symmetry $S_{h}$, we obtain Assertion 2 .

Finally, Assertion 2 and (24) give us Assertion 3.

The Main Theorem is a consequence of the following results: Propositions 1, 5, and 6, Lemmas 7, 8, and 9, Remark 2, Theorem 4 in [10] and Theorem 3.32 in $[\mathbf{9}]$.

Acknowledgements. We would like to thank Professor F.J. López for helpful conversations.

\section{References}

[1] R.B. Burckel, An Introduction to Classical Analysis, Vol. 1, Birkhäuser, Basel, 1979, MR 0555733, Zbl 0434.30001.

[2] S.Y. Cheng, Eigenfunctions and nodal sets, Comment. Math. Helvetici, 51 (1976), 43-55, MR 0397805, Zbl 0334.35022.

[3] E.A. Coddington and N. Levinson, Theory of Ordinary Differential Equations, McGraw-Hill Book Company, New York, 1955, MR 0069338, Zbl 0064.33002.

[4] P. Collin, R. Kusner, W.H. Meeks and H. Rosenberg, The topology, geometry and conformal structures of properly embedded minimal surfaces. Preprint.

[5] L. Ferrer and F. Martín, Minimal discs bounded by straight lines, Differential Geometry, Valencia, 2001, 157-166, World Sci. Publishing, River Edge, NJ, 2002, MR 1922045.

[6] H. Jenkins and J. Serrin, Variational problems of minimal surface type. II. Boundary value problems for the minimal surface equation, Arch. Rat. Mech. Anal., 21 (1966), 321-342, MR 0190811, Zbl 0171.08301. 
[7] H. Karcher, Construction of Minimal Surfaces, Surveys in Geometry 1989/90, University of Tokyo, 1989; Also: Vorlesungsreihe Nr. 1, SFB 256, Bonn, 1989.

[8] F.J. López, Minimal surfaces in a cone, Ann. Global Anal. Geom., 20(3) (2001), 253-299, MR 1866418, Zbl 0998.53041.

[9] F.J. López and F. Martín, A uniqueness theorem for properly embedded minimal surfaces bounded by straight lines, J. Austr. Math. Soc. (Series A), 69 (2000), 362402, MR 1793470, Zbl 0997.53004.

[10] _ Minimal surfaces in a wedge of a slab, Comm. Anal. Geom., 9(4) (2001), 683-723, MR 1868918.

[11] F.J. López and F. Wei, Properly immersed minimal disks bounded by straight lines, Math. Ann., 318 (2000), 667-706, MR 1802506, Zbl 0994.53004.

[12] W.H. Meeks and H. Rosenberg, The geometry and conformal structure of properly embedded minimal surfaces of finite topology in $\mathbb{R}^{3}$, Invent. Math., 114 (1993), 625639, MR 1244914, Zbl 0803.53007.

[13] J.C.C. Nitsche, Lectures on Minimal Surfaces, Volume 1, Cambridge University Press, Cambridge, 1989, MR 1015936, Zbl 0688.53001.

[14] R. Schoen, Uniqueness, symmetry and embeddedness of minimal surfaces, J. Differential Geom., 18 (1983), 791-809, MR 0730928, Zbl 0575.53037.

[15] M. Weber, The genus one helicoid is embedded, Habilitationsschrift, Bonn, 1999.

Received November 6, 2002. This research was partially supported by MCYT-FEDER Grant number BFM2001-3489.

Departamento de Geometría y Topología

UNIVERSIDAD DE GRANADA

18071 GRANADA

SPAIN

E-mail address: lferrer@ugr.es

Departamento de Geometría y Topología

UNIVERSIDAD DE GRANADA

18071 GRANADA

SPAIN

E-mail address: fmartin@ugr.es 